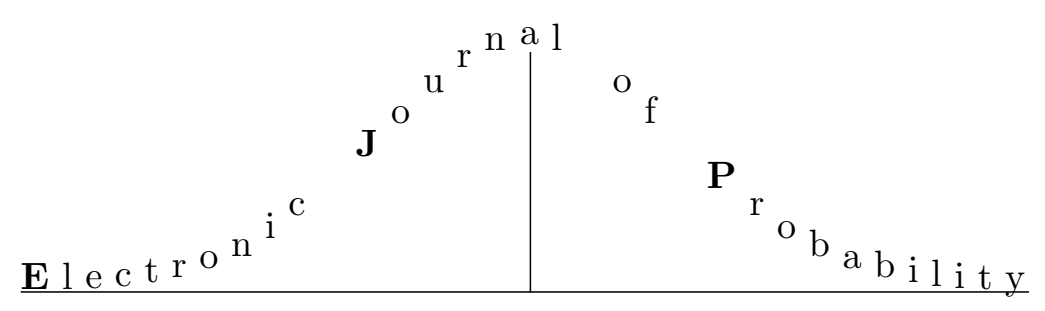

Vol. 15 (2010), Paper no. 71, pages 2163-2199.

Journal URL

http://www.math.washington.edu/ ejpecp/

\title{
G distributions and the beta-gamma algebra
}

\author{
Daniel Dufresne \\ Centre for Actuarial Studies \\ University of Melbourne \\ Melbourne VIC 3010, Australia \\ dufresne@unimelb.edu.au
}

\begin{abstract}
.
This paper has four interrelated themes: (1) express Laplace and Mellin transforms of sums of positive random variables in terms of the Mellin transform of the summands; (2) show the equivalence of the two Barnes' lemmas with known properties of gamma distributions; (3) establish properties of the sum of two reciprocal gamma variables, and related results; (4) study the G distributions (whose Mellin transforms are ratios of products of gamma functions).
\end{abstract}

Key words: Beta distribution; beta product distribution; gamma distribution; G distributions; Barnes' lemmas; Mellin transforms; infinite divisibility; Macdonald's function.

AMS 2010 Classification: 97K60, 65R10, 60E07, 33B15.

Submitted to EJP on May 25, 2010, final version accepted November 12, 2010. 


\section{Introduction}

Sections 2 to 5 derive and then apply formulas for Laplace and Mellin transforms of probability distributions on $\mathbb{R}_{+}$. The specific applications given relate to properties of the beta and gamma distributions, the so-called "beta-gamma algebra". The best-known of those properties are probably

$$
G_{1}^{(a)}+G_{2}^{(b) \stackrel{\mathrm{d}}{=}} G_{3}^{(a+b)}, \quad B^{(a, b)} G_{4}^{(a+b)} \stackrel{\mathrm{d}}{=} G_{5}^{(a)} .
$$

(Here and in the sequel, "d = means "has the same distribution as", the symbol " $G_{j}^{(\alpha)}$ " denotes a $\operatorname{Gamma}(\alpha, 1)$ distributed variable, while $B^{(\alpha, \beta)}$ denotes a variable with a $\operatorname{Beta}(\alpha, \beta)$ distribution; the variables on the left-hand sides of the identities in (1.1) are independent.) Other properties of beta and gamma distributions are recalled or obtained in Sections 4 and 5 of this paper. It will be shown that Barnes' first lemma (Barnes, 1908) and second lemma (Barnes, 1910) are each equivalent to a property of beta and gamma variables. This connects the properties of beta and gamma distributions with classical analysis.

Section 2 gives formulas expressing the Laplace transform of a probability distribution in terms of its Mellin transform. Section 3 gives formulas for the Mellin transform of a sum of positive variables in terms of the joint Mellin transform of the variables. These formulas have obvious counterparts for Mellin transforms of ordinary functions. For instance, Theorem 3.1 may be rephrased as follows for $K=2$. Let $f_{1}, f_{2}$ be functions on $\mathbb{R}_{+}$, with Mellin transforms

$$
\mathcal{M} f_{j}(s)=\int_{0}^{\infty} d x x^{s-1} f_{j}(x), \quad j=1,2 .
$$

Let $g=f_{1} * f_{2}$ be the additive convolution

$$
g(x)=\int_{0}^{x} d y f_{1}(y) f_{2}(x-y) .
$$

Theorem 3.1 says that if $\Re(p)>c>0$ and

$$
\int_{0}^{\infty} d x x^{-c}\left|f_{1}(x)\right|<\infty, \quad \int_{0}^{\infty} d x x^{c-\Re(p)}\left|f_{2}(x)\right|<\infty
$$

then

$$
\mathcal{M} g(1-p)=\frac{1}{2 \pi i} \int_{c-i \infty}^{c+i \infty} d z\left(\mathcal{M} f_{1}\right)(1-z)\left(\mathcal{M} f_{2}\right)(1+z-p) \frac{\Gamma(z) \Gamma(p-z)}{\Gamma(p)}
$$

This author has not found the last formula in the literature, though it is not far from formula (2.4.7), p.88, in Andrews et al. (2000), and is also close to the formula in Exercise 36 on page 121 of the same book. Whether or not it is already in the literature, Theorem 3.1 does not appear to have been used in probability theory before. For instance, Chamayou \& Letac (1999, p.1053) are precisely asking for the Mellin transform of the additive convolution of two probability densities, as we briefly explain. Denote $\mathbf{G}\left(a_{1}, \ldots, a_{K} ; b_{1}, \ldots, b_{N}\right)$ the probability distribution, if it exists, that has Mellin transform

$$
\int_{0}^{\infty} x^{p} G\left(a_{1}, \ldots, a_{K} ; b_{1}, \ldots, b_{N} ; d x\right)=\frac{\Gamma\left(a_{1}+p\right) \cdots \Gamma\left(a_{K}+p\right)}{\Gamma\left(a_{1}\right) \cdots \Gamma\left(a_{K}\right)} \frac{\Gamma\left(b_{1}\right) \cdots \Gamma\left(b_{N}\right)}{\Gamma\left(b_{1}+p\right) \cdots \Gamma\left(b_{N}+p\right)} .
$$


In their Proposition 7, Chamayou and Letac let $X \sim \mathbf{G}\left(a_{1}, \ldots, a_{K+1} ; b_{1}, \ldots, b_{K}\right)$ and $Y \sim$ $\operatorname{Gamma}(c, 1)$ be independent, and then give an expression for $\mathrm{E}(X+Y)^{n}, n=1,2, \ldots$, in terms of hypergeometric functions. They then comment (p.1053): "There is a little challenge behind the previous proposition. Can we extend the result to the computation of the Mellin transform $\mathrm{E}(X+Y)^{s}$ ?" Theorem 3.1 gives an answer to that question: for $0<c_{0}<\min \left(a_{1}, \ldots, a_{K+1}\right)$, $c_{0}<\Re(p)<c_{0}+c$,

$$
\begin{aligned}
\mathrm{E}(X+Y)^{-p}= & \frac{\Gamma\left(b_{1}\right) \cdots \Gamma\left(b_{K}\right)}{\Gamma\left(a_{1}\right) \cdots \Gamma\left(a_{K+1}\right)} \\
& \quad \times \frac{1}{2 \pi i} \int_{c_{0}-i \infty}^{c_{0}+i \infty} d z \frac{\Gamma\left(a_{1}-z\right) \cdots \Gamma\left(a_{K+1}-z\right) \Gamma(c+z-p)}{\Gamma\left(b_{1}-z\right) \cdots \Gamma\left(b_{K}-z\right)} \frac{\Gamma(z) \Gamma(p-z)}{\Gamma(p)} .
\end{aligned}
$$

Integrals of ratios of products of gamma functions, such as the one above, are called "Barnes integrals" (or "Mellin-Barnes integrals"). Barnes $(1908,1910)$ found simplified expressions for two such integrals, and those identities have come to be known as Barnes' first and second lemmas. Section 4 shows that some special cases (involving beta and gamma distributions) where the explicit distribution of $X+Y$ is known are in fact equivalent to Barnes' first and second lemmas. Barnes' lemmas thus have probabilistic interpretations, or, taking the opposite point of view, those lemmas are given probabilistic proofs, in terms of relatively simple properties of beta and gamma distributions. Theorem 3.2 is an extension of the binomial formula for $\mathrm{E}(X+Y)^{r}$, for $r \notin \mathbb{N}$.

A probabilistic interpretation of (1.3) is that if $X$ has the Mellin transform on the right for positive $\left(a_{k}, b_{n}\right)$, then, introducing independent gamma variables, $X$ satisfies the identity

$$
G_{1}^{\left(a_{1}\right)} \cdots G_{K}^{\left(a_{K}\right)} \stackrel{\mathrm{d}}{=} X G_{1}^{\left(b_{1}\right)} \cdots G_{N}^{\left(b_{N}\right)} .
$$

This is an extension of the second identity in (1.1).

The Mellin transform of $X+Y$ has relevance in solving the stochastic equation

$$
X \stackrel{\mathrm{d}}{=} A(X+Y),
$$

and this is noted in Section 5; solving these equations is directly related to finding the distribution of

$$
\sum A_{1} \cdots A_{k} Y_{k},
$$

a topic that has been studied for several decades; Vervaat (1979) is a classical reference, another one is Dufresne (1998). In the same section, formulas for the distribution of the sum of two independent reciprocal gamma variables are given, as well as other related results; these are consequences of Barnes' first lemma. One problem is to find versions of the identities in (1.1) with the gamma variables replaced by their reciprocals; specifically: (1) what can be said about the distribution of $1 / G^{(a)}+1 / G^{(b)}$ ?; $(2)$ is it possible to find the distribution for a random variable $A$, independent of the others, with the property that

$$
\frac{1}{G^{(a)}} \stackrel{\mathrm{d}}{=} A\left(\frac{1}{G_{1}^{(a)}}+\frac{1}{G_{2}^{(b)}}\right) ?
$$

A related identity (from Chaumont \& Yor, 2003) is briefly studied. Connections with the Bessel functions $K_{\nu}(z)$ (called Macdonald's functions) are also noted. 
The beta product distribution was defined in a recent paper (Dufresne, 2010) and also occurs in Section 5. It is a G distribution of type (1.3) with $K=N=2$, and has support $(0,1)$. In Section 6 , the beta product distribution is extended to include cases where the support is $(0,1]$ (there is a point mass at $u=1$ ). This naturally leads to a more general study of the $G$ distributions "of order $(N, N)$ ", that is, of those that satisfy (1.3) with $K=N$. It is shown that if (1.3) holds for all $p \geq 0$, then the distribution's support is necessarily a subset of $(0,1]$, with a point mass at $u=1$ if $\sum_{k=1}^{K} a_{k}=\sum_{n=1}^{N} b_{n}$. The $\mathrm{G}$ distributions of order $(N, N)$ strictly include all product convolutions of ordinary beta distributions, and it is then interesting to ask whether they have the same infinite divisibility property, as it is known that $\log B^{(a, b)}$ is infinitely divisible. (A reference on infinite divisibility is Bondesson (1992).) It is shown in Section 6 that for G distributions of order $(N, N)$ the log transformed law is always infinitely divisible if $N=1$ or 2 , but that it may or may not be if $N \geq 3$; examples of both possibilities are given. It is also shown that this family of $\log$ transformed $G$ distributions includes all rational distributions on $\mathbb{R}_{+}$(Cox, 1955), and that for a subclass there is an explicit decomposition in terms of rational distributions. A corollary is that the law of $\log \left(\frac{1}{G^{(a)}}+\frac{1}{G^{(b)}}\right)$ is infinitely divisible. The distributions $G^{(K, N)}$ with $K>N$ are also considered. In particular, it is shown that the law of the product $G^{\left(a_{1}\right)} \cdots G^{\left(a_{K}\right)}$ of $K \geq 3$ gamma variables makes sense when some of the parameters $a_{1}, \ldots, a_{K}$ are complex.

Note that, contrary to Chamayou, Letac and possibly all previous authors, we allow the parameters $a_{k}, b_{n}$ of $\mathrm{G}$ distributions to be complex numbers.

Here is some of the notation used. A random variable with a $\operatorname{Gamma}(\alpha, 1)$ distribution is denoted $G^{(\alpha)}$; similarly, $B^{(\alpha, \beta)}$ has a $\operatorname{Beta}(\alpha, \beta)$ distribution. Subscripts are added when required. We define $(a)_{q}=\Gamma(a+q) / \Gamma(a)$ for any $a, q \in \mathbb{C},-a-q \notin \mathbb{N}$ (this is called the "shifted factorial" or "Pochhammer symbol" when $n \in \mathbb{N})$. The duplication and reflection formulas for the gamma function are used:

$$
\Gamma(2 z) \Gamma\left(\frac{1}{2}\right)=2^{2 z-1} \Gamma(z) \Gamma\left(z+\frac{1}{2}\right) ; \quad \Gamma(z) \Gamma(1-z)=\frac{\pi}{\sin \pi z} .
$$

It is often more convenient to use the following equivalent form of the duplication formula:

$$
(2 z)_{2 p}=4^{p}(z)_{p}\left(z+\frac{1}{2}\right)_{p} .
$$

(Just let $z=\frac{1}{2}$ to obtain the usual form of the duplication formula.) The Appendix shows that the duplication formula can be obtained from properties of beta and gamma distributions, to which it is in fact equivalent. The same line of attack gives a partial justification for the Gauss multiplication formula.

The Mellin transform of a probability distribution $\mu$ on $\mathbb{R}_{+}$(or of a random variable $X$ with that distribution) will be defined as the mapping

$$
p \mapsto \int_{\mathbb{R}_{+}} x^{p} d \mu(x)=\mathrm{E} X^{p},
$$

as in (1.3). This is different from the definition used in analysis (see the exponent " $s-1$ " in (1.2)). A convenient summary of results on Mellin transforms is found in Appendix 2 of Patterson (1995).

\section{Laplace transform of sums of positive variables}

The identity

$$
e^{-x}=\frac{1}{2 \pi i} \int_{\ell_{c}} d z x^{-z} \Gamma(z)
$$


is valid for $\Re(x)>0, c>0$, if the path of integration is:

$$
\ell_{c}=\{c+i w \mid-\infty<w<\infty\}
$$

Formula (2.1) may be obtained by summing residues, or it can be seen as an inverse Mellin transform (a proof is given in Andrews et al., 2000, p.85).

Theorem 2.1. Suppose $c_{j}>0, \Re\left(s_{j}\right)>0, X_{j}>0$ for $j=1, \ldots, K$, and $\mathrm{E}\left(X_{1}^{-c_{1}} \cdots X_{j}^{-c_{K}}\right)<\infty$. Then

$\mathrm{E} \exp \left(-\sum_{j=1}^{K} s_{j} X_{j}\right)=\frac{1}{(2 \pi i)^{K}} \int_{\ell_{c_{1}}} d z_{1} \cdots \int_{\ell_{c_{K}}} d z_{K} s_{1}^{-z_{1}} \cdots s_{K}^{-z_{K}} \mathrm{E}\left(X_{1}^{-z_{1}} \cdots X_{K}^{-z_{K}}\right) \Gamma\left(z_{1}\right) \cdots \Gamma\left(z_{K}\right)$.

Proof. We will use the following result (for a proof, see Andrews et al. (2000), p.21).

Lemma 2.2. If $z=v+i w, a_{1} \leq v \leq a_{2}, v, w \in \mathbb{R}$ and $|w| \rightarrow \infty$, then

$$
|\Gamma(v+i w)|=\sqrt{2 \pi}|w|^{v-\frac{1}{2}} e^{-\pi|w| / 2}[1+\mathcal{O}(1 /|w|)],
$$

where the constant implied by "OO' depends only on $a_{1}$ and $a_{2}$.

Insert $x=s_{j} X_{j}$ in (2.1) for $j=1, \ldots, K$, and multiply the resulting identities together. Take expectations on both sides; the multiple integral so obtained will be shown to converge absolutely. First, $\left|\mathrm{E}\left(X_{1}^{-z_{1}} \cdots X_{j}^{-z_{K}}\right)\right|$ is uniformly bounded by $\mathrm{E}\left(X_{1}^{-c_{1}} \cdots X_{j}^{-c_{K}}\right)$ on $\ell_{c_{1}} \times \cdots \times \ell_{c_{K}}$. Second, if $z_{j}=c_{j}+i w_{j}, \arg s_{j}=\theta_{j},-\frac{\pi}{2}<\theta_{j}<\frac{\pi}{2}$,

$$
\left|s_{j}^{-z_{j}} \Gamma\left(z_{j}\right)\right|=\sqrt{2 \pi}\left|s_{j}\right|^{-c_{j}}|w|^{c_{j}-\frac{1}{2}} e^{\theta_{j} w_{j}-\pi\left|w_{j}\right| / 2}\left[1+\mathcal{O}\left(1 /\left|w_{j}\right|\right)\right] .
$$

The function $s_{1}^{-z_{1}} \cdots s_{K}^{-z_{K}} \mathrm{E}\left(X_{1}^{-z_{1}} \cdots X_{j}^{-z_{K}}\right) \Gamma\left(z_{1}\right) \cdots \Gamma\left(z_{K}\right)$ is then absolutely integrable over $\ell_{c_{1}} \times$ $\cdots \times \ell_{c_{K}}$.

A simple application of Theorem 2.1 is the case where $X \sim \operatorname{Gamma}(a, 1)$, which reads

$$
(1+s)^{-a}=\frac{1}{2 \pi i} \int_{\ell_{c}} d z s^{-z} \frac{\Gamma(a-z) \Gamma(z)}{\Gamma(a)}, \quad 0<c<a, \Re(s)>0 .
$$

This is also an inverse Mellin transform, or else it can be seen as a particular case of Theorem 3.1 below.

Several questions are raised by Theorem 2.1: (1) What if $\mathrm{P}\left(X_{j}=0\right)>0$ ? (2) Can the constraint $\Re\left(s_{j}\right)>0$ be weakened? (3) What if $\mathrm{E} X_{j}^{-c_{j}}=\infty$ for all $c_{j}>0$ ? (4) Can the path of integration $\ell_{c_{j}}$ be moved to the left of the origin? The rest of this section answers these questions in turn. For ease of presentation, we study these questions in the case of one variable only.

Regarding the first question, all formulas may be adapted to the case $X \geq 0$ by writing

$$
\mathrm{E} e^{-s X}=\mathrm{P}(X=0)+\mathrm{P}(X>0) \mathrm{E} e^{-s Y},
$$

where $Y$ has the conditional distribution of $X$, given $X>0$. As to the second question, in many cases the Laplace transform has an analytic continuation beyond the region where it exists, so not 
surprisingly the formula in Theorem 2.1 holds, under some conditions, for the analytic continuation of the Laplace transform. To fix ideas, the Laplace transform of the $\operatorname{Gamma}(a, 1)$ distribution is $(1+s)^{-a}$ for $\Re(s)>-1$, but the function $(1+s)^{-a}$ has an analytic continuation in $\mathbb{C}-(-\infty,-1]$. In order to distinguish between the two functions in the corollary below we write $\mathcal{L}_{X}(s)$ for the transform together with its analytic continuation.

The next result answers the third question.

Corollary 2.3. Suppose $X>0, c>0, \mathrm{E} X^{-c}<\infty,-\pi<\theta<\pi$, and that

$$
\int_{0}^{\infty} d w w^{c-\frac{1}{2}}\left|\mathrm{E} X^{-c-i w}\right| e^{w\left(|\theta|-\frac{\pi}{2}\right)}<\infty
$$

Then, for any $s=r e^{i \theta}, r>0$,

$$
\mathcal{L}_{X}(s)=\frac{1}{2 \pi i} \int_{\ell_{c}} d z s^{-z} \mathrm{E} X^{-z} \Gamma(z)
$$

Proof. The right-hand side of the last equation is analytic for $s$ in an open set as soon as the integral converges absolutely on the closure of that set. If we write $z=c+i w$, then (Lemma 2.2):

$$
\left|s^{-z} \mathrm{E} X^{-z} \Gamma(z)\right|=|s|^{-c} e^{\theta w}\left|\mathrm{E} X^{-c-i w}\right| \sqrt{2 \pi} w^{c-\frac{1}{2}} e^{-|w| \pi / 2}[1+\mathcal{O}(1 /|w|)] .
$$

Corollary 2.4. Suppose $X>0, s>0$. (a) Then

$$
\mathrm{E} e^{-s X}=\frac{1}{2}+\frac{1}{2 \pi} \lim _{\epsilon \downarrow 0} \int_{\epsilon}^{\infty} d w\left[s^{-i w} \mathrm{E} X^{-i w} \Gamma(i w)+s^{i w} \mathrm{E} X^{i w} \Gamma(-i w)\right]
$$

(b) If $\mathrm{E}|\log X|<\infty$, then the integral above converges absolutely (so the limit may be removed).

Proof. Recall formula (2.1) with $c, \Re(x)>0$ and let $0<\epsilon<R<\infty$. Let $z=v+i w$ and consider the contour consisting of the segments

$$
\begin{aligned}
& S_{1, \epsilon, R}=\{v=0, \epsilon<w<R\}, S_{2, R}=\{w=R, 0 \leq v \leq c\}, S_{3, R}=\{v=c,-R<w<R\} \\
& S_{4, R}=\{w=-R, 0 \leq v \leq c\}, S_{5, \epsilon, R}=\{v=0,-R<w<-\epsilon\}
\end{aligned}
$$

and the semi-circle $C_{\epsilon}=\left\{z=\epsilon e^{i \theta},-\frac{\pi}{2} \leq \theta \leq \frac{\pi}{2}\right\}$. The function $x^{-z} \Gamma(z)$ is analytic inside the contour, and extends continuously to the contour; thus its integral over the contour is zero. The integral over $S_{2, R}$ and $S_{4, R}$ tends to zero as $R$ tends to infinity, from Lemma 2.2. Hence,

$$
e^{-x}=\frac{1}{2 \pi i} \int_{S_{1, \epsilon, \infty} \cup S_{5, \epsilon, \infty} \cup C_{\epsilon}} d z x^{-z} \Gamma(z)
$$

The integral over $C_{\epsilon}$ equals

$$
\frac{1}{2 \pi} \int_{-\frac{\pi}{2}}^{\frac{\pi}{2}} d \phi x^{-\epsilon e^{i \phi}} \Gamma\left(1+\epsilon e^{i \phi}\right) \rightarrow \frac{1}{2}
$$


as $\epsilon$ tends to 0 . Thus,

$$
e^{-x}=\frac{1}{2}+\frac{1}{2 \pi} \int_{0}^{\infty} d w\left[x^{-i w} \Gamma(i w)+x^{i w} \Gamma(-i w)\right] .
$$

Next, replace $x$ with $s X$, with $\Re(s)>0$. Then

$$
\mathrm{E} e^{-s X}=\frac{1}{2}+\frac{1}{2 \pi}\left(\mathrm{E} I_{1}+\mathrm{E} I_{2}\right),
$$

where

$$
\begin{aligned}
& I_{1}=\int_{1}^{\infty} d w\left[(s X)^{-i w} \Gamma(i w)+(s X)^{i w} \Gamma(-i w)\right] \\
& I_{2}=\int_{0}^{1} d w\left[(s X)^{-i w} \Gamma(i w)+(s X)^{i w} \Gamma(-i w)\right] .
\end{aligned}
$$

In the case of $\mathrm{E} I_{1}$, the order of integration and expectation may be reversed by dominated convergence. As to $I_{2}$, write

$$
\begin{aligned}
(s X)^{-i w} \Gamma(i w)+(s X)^{i w} \Gamma(-i w) & =\frac{1}{i w}\left[(s X)^{-i w} \Gamma(1+i w)-(s X)^{i w} \Gamma(1-i w)\right] \\
& =\frac{2}{w} \int_{0}^{\infty} d t e^{-t} \sin \left(w \log \frac{t}{s X}\right)
\end{aligned}
$$

and then

$$
I_{2}=2 \int_{0}^{1} \frac{d w}{w} \int_{0}^{\infty} d t e^{-t} \sin \left(w \log \frac{t}{s X}\right) .
$$

Since $|\sin (x) / x|$ is uniformly bounded for real $x$, the above double integral converges absolutely for any fixed $X>0$, and the order of integration may be reversed. Thus,

$$
\mathrm{E} I_{2}=2 \mathrm{E} \int_{0}^{1} d w \frac{\sin (w Y)}{w}=2 \mathrm{E} \int_{0}^{Y} d v \frac{\sin v}{v},
$$

where $Y=\log \left[G^{(1)} /(s X)\right], G^{(1)}$ independent of $X$. This expectation is finite, since $\left|\int_{0}^{y} d v \sin v / v\right|$ is uniformly bounded for $y \in \mathbb{R}$. For the same reason,

$$
\mathrm{E} I_{2}=2 \lim _{\epsilon \downarrow 0} \mathrm{E} \int_{\epsilon}^{1} d w \frac{\sin (w Y)}{w}
$$

which is in turn equal to

$$
2 \lim _{\epsilon \downarrow 0} \int_{\epsilon}^{1} \frac{d w}{w} \mathrm{E} \sin (w Y)
$$

by dominated convergence. This yields part (a). For part (b), if $\mathrm{E}|\log X|<\infty$, then $\mathrm{E}|Y|<\infty$ and

$$
\left|\mathrm{E} \frac{\sin (w Y)}{w}\right| \leq \mathrm{E}|Y|, \quad w \in \mathbb{R}
$$

which implies that $\int_{0}^{1} \frac{d w}{w} \mathrm{E} \sin (w Y)$ converges absolutely. 
Observe that the condition that $\mathrm{E}|\log X|$ be finite is not necessary for absolute convergence of the integral in Corollary 2.4. For instance, if $\log X$ has a symmetric stable distribution with characteristic function $\exp \left(-a|w|^{\alpha}\right), 0<\alpha<1$, then $\mathrm{E}|\log X|$ is infinite, but

$$
\mathrm{E} X^{i w} \sim 1+\mathcal{O}\left(|w|^{\alpha}\right), \quad w \rightarrow 0
$$

and thus $\mathrm{E} \sin (w Y) / w=\mathcal{O}\left(|w|^{\alpha-1}\right)$ is integrable over $(0,1)$.

The fourth question was about moving the path of integration to the left in Theorem 2.1.

Corollary 2.5. If $X>0$, E $X^{q}<\infty$ for some $q>0, q \notin \mathbb{N}$, then for $\Re(s)>0$ :

$$
\mathrm{E} e^{-s X}=\sum_{k=0}^{[q]} \frac{(-s)^{k} \mathrm{E} X^{k}}{k !}+\frac{1}{2 \pi i} \int_{\ell_{-q}} d z s^{-z} \mathrm{E} X^{-z} \Gamma(z)
$$

Proof. The result follows from a modification of (2.1):

$$
e^{-x}=\sum_{j=0}^{n} \frac{(-x)^{j}}{j !}+\frac{1}{2 \pi i} \int_{\ell_{-q}} d z x^{-z} \Gamma(z)
$$

where $\Re(x)>0$ and $n<q<n+1$. That formula may be proved by summing residues, or else by deforming the path of integration while forming a circle around each singularity $z=0,-1, \ldots$..

Note that Eq.(2.2) gives an alternative expression for the remainder term of the Taylor expansion for $e^{-x}$ : for $n=0,1, \ldots$ and $0<\epsilon<1$,

$$
\frac{(-1)^{n+1}}{n !} \int_{0}^{x} d y(x-y)^{n} e^{-y}=\frac{1}{2 \pi i} \int_{\ell_{-n-\epsilon}} d z x^{-z} \Gamma(z) .
$$

\section{Mellin transforms of sums of positive variables}

Theorem 3.1. Suppose $c_{j}>0, j=1, \ldots, K-1, X_{j}>0, j=1, \ldots, K, \Re(p)>c_{1}+\ldots+c_{K-1}$ and $\mathrm{E}\left(X_{1}^{-c_{1}} \cdots X_{K-1}^{-c_{K}-1} X_{K}^{c_{1}+\cdots+c_{K-1}-\Re(p)}\right)<\infty$. Then

$$
\begin{aligned}
\mathrm{E}\left(X_{1}+\cdots+X_{K}\right)^{-p}= & \frac{1}{(2 \pi i)^{K-1}} \int_{\ell_{c_{1}}} d z_{1} \cdots \int_{\ell_{c_{K-1}}} d z_{K-1} \\
& \mathrm{E}\left(X_{1}^{-z_{1}} \cdots X_{K-1}^{-z_{K-1}} X_{K}^{z_{1}+\cdots+z_{K-1}-p}\right) B\left(z_{1}, \ldots, z_{K-1}, p-z_{1}-\ldots-z_{K-1}\right)
\end{aligned}
$$

(which is finite), where

$$
B\left(a_{1}, \ldots, a_{K}\right)=\frac{\Gamma\left(a_{1}\right) \cdots \Gamma\left(a_{K}\right)}{\Gamma\left(a_{1}+\ldots+a_{K}\right)}, \quad \Re\left(a_{j}\right)>0, j=1, \ldots, K
$$

is the Dirichlet function. 
Proof. The finiteness of the expectation is proved as follows: if $q=\Re(p)$, then

$$
\left(X_{1}+\cdots+X_{K}\right)^{-q} \leq \frac{X_{1}^{-c_{1}} \cdots X_{K-1}^{-c_{K}-1}}{\left(X_{1}+\cdots+X_{K}\right)^{q-c_{1}-\cdots-c_{K-1}}} .
$$

From Theorem 2.1, if $\Re\left(x_{j}\right)>0, j=1, \ldots, K-1, s>0$, then

$$
\begin{aligned}
\exp ( & \left.-s \sum_{j=1}^{K-1} x_{j}\right) \\
& =\frac{1}{(2 \pi i)^{K-1}} \int_{\ell_{c_{1}}} d z_{1} \cdots \int_{\ell_{c_{K-1}}} d z_{K-1} s^{-z_{1}-\cdots-z_{K-1}} x_{1}^{-z_{1}} \cdots x_{K-1}^{-z_{K-1}} \Gamma\left(z_{1}\right) \cdots \Gamma\left(z_{K-1}\right) .
\end{aligned}
$$

Suppose $\Re\left(x_{K}\right)>0, \Re(p)>c_{1}+\ldots+c_{K-1}$, multiply both sides by $s^{p-1} e^{-s x_{K}} / \Gamma(p)$ and integrate with respect to $s \in(0, \infty)$. By Fubini's theorem, the order of integration on the right-hand side may be changed at will, and

$$
\begin{aligned}
\left(x_{1}+\cdots+x_{K}\right)^{-p}=\frac{1}{(2 \pi i)^{K-1}} \int_{\ell_{c_{1}}} d z_{1} \cdots \int_{\ell_{c_{K-1}}} d z_{K-1} x_{1}^{-z_{1}} \cdots x_{K-1}^{-z_{K-1}} x_{K}^{z_{1}+\cdots+z_{K-1}-p} \\
\times \\
\times\left(z_{1}, \ldots, z_{K-1}, p-z_{1}-\cdots-z_{K-1}\right) .
\end{aligned}
$$

Replace $x_{j}$ with $X_{j}, j=1, \ldots, K$, and then take expectations on both sides. Expectation and integration commute by Lemma 2.2 and Fubini's theorem.

This theorem has immediate applications to gamma distributions, see Sections 4 and 5 .

Theorem 3.2. Suppose $X, Y>0, q>0, q \notin \mathbb{N}, \Re(p)>-q$ and

$$
\mathrm{E} Y^{-\Re(p)}<\infty, \quad \mathrm{E}\left(X^{q} Y^{-q-\Re(p)}\right)<\infty .
$$

Then

$$
\mathrm{E}(X+Y)^{-p}=\sum_{j=0}^{[q]} \frac{(-1)^{j}(p)_{j} \mathrm{E}\left(X^{j} Y^{-p-j}\right)}{j !}+\frac{1}{2 \pi i} \int_{\ell_{-q}} d z \mathrm{E}\left(X^{-z} Y^{z-p}\right) B(z, p-z)
$$

Proof. From (2.2), for $s, x>0$,

$$
e^{-s x}=\sum_{j=0}^{[q]} \frac{(-s x)^{j}}{j !}+\frac{1}{2 \pi i} \int_{\ell_{-q}} d z(s x)^{-z} \Gamma(z)
$$

Multiply by $s^{\zeta-1} e^{-s y} / \Gamma(\zeta)$ for $\Re(\zeta), y>0$, and integrate with respect to $s \in(0, \infty)$ to get:

$$
(x+y)^{-\zeta}=\sum_{j=0}^{[q]} \frac{(-1)^{j}(\zeta)_{j} x^{j} y^{-\zeta-j}}{j !}+\frac{1}{2 \pi i} \int_{\ell_{-q}} d z x^{-z} y^{z-\zeta} B(z, \zeta-z) .
$$


Both sides of this equation are seen to be analytic for $\Re(\zeta)>-q$, and the identity then holds in that larger region. Replace $\zeta, x, y$ with $p, X, Y$, and then take expectations to finish the proof. This is possible because, under the stated assumptions,

$$
\mathrm{E} X^{r} Y^{-\Re(p)-r}=\mathrm{E} Y^{-\Re(p)}\left(\frac{X}{Y}\right)^{r} \leq \mathrm{E} Y^{-\Re(p)}\left[1+\left(\frac{X}{Y}\right)^{q}\right]<\infty, \quad 0 \leq r \leq q .
$$

Moreover, by Lemma 2.2 and dominated convergence, the integral in the formula converges absolutely. Finally,

$$
\mathrm{E}(X+Y)^{-\Re(p)} \leq C_{p}\left(\mathrm{E} X^{-\Re(p)}+\mathrm{E} Y^{-\Re(p)}\right)<\infty
$$

where $C_{p}=2^{-\Re(p)}$ if $-q<\Re(p) \leq 0, C_{p}=\frac{1}{2}$ if $\Re(p)>0$.

When $-p=n \in \mathbb{N}$, the formula in Theorem 3.2 boils down to the familiar binomial sum. Formula (3.1) is a rewriting of the Taylor expansion of $(x+y)^{-p}$ about $x=0$. Turning the formula around and setting $x=y=1$ yields

$$
\frac{1}{2 \pi i} \int_{\ell_{-q}} d z B(z, p-z)=2^{-p}-\sum_{j=0}^{[q]} \frac{(-1)^{j}(p)_{j}}{j !} .
$$

\section{Barnes' lemmas and the beta-gamma algebra}

The two Barnes' lemmas will be shown to be consequences of part (a) of Theorem 4.2, which is itself equivalent to the well-known property (4.1) below (more on this equivalence at the end of this section). We first state the two Barnes' lemmas. The original references are given, but see Andrews et al. (2000, pp. 89-91) for more details; here $A, B, C, D$ are any complex constants such that the path of integration described can be drawn.

(1) (Barnes, 1908) For a suitably curved line of integration, so that the decreasing sequences of poles lie to the left and the increasing sequences lie to the right of the contour,

$$
\frac{1}{2 \pi i} \int_{-i \infty}^{i \infty} d z \Gamma(A+z) \Gamma(B+z) \Gamma(C-z) \Gamma(D-z)=\frac{\Gamma(A+C) \Gamma(A+D) \Gamma(B+C) \Gamma(B+D)}{\Gamma(A+B+C+D)} .
$$

(2) (Barnes, 1910) For a suitably curved line of integration, so that the decreasing sequences of poles lie to the left and the increasing sequences lie to the right of the contour, if $E=A+B+C+D$,

$$
\begin{aligned}
\frac{1}{2 \pi i} \int_{-i \infty}^{i \infty} d z \frac{\Gamma(A+z) \Gamma(B+z) \Gamma(C+z) \Gamma(D-z) \Gamma(-z)}{\Gamma(E+z)} & \\
= & \frac{\Gamma(A) \Gamma(B) \Gamma(C) \Gamma(A+D) \Gamma(B+D) \Gamma(C+D)}{\Gamma(E-A) \Gamma(E-B) \Gamma(E-C)} .
\end{aligned}
$$

Theorem 4.1. Barnes' first lemma is equivalent to the additivity property of gamma distributions: if $a, b>0$ and $G_{1}^{(a)}, G_{2}^{(b)}$ are independent, then $G_{1}^{(a)}+G_{2}^{(b)} \stackrel{\mathrm{d}}{=} G_{3}^{(a+b)}$. 
Proof. From Theorem 3.1, the additivity property of gamma variables may be written as:

$$
\mathrm{E}\left(G_{1}^{(a)}+G_{2}^{(b)}\right)^{-p}=\frac{1}{2 \pi i} \int_{\ell_{c}} d z \mathrm{E}\left[\left(G_{1}^{(a)}\right)^{-z}\left(G_{2}^{(b)}\right)^{z-p}\right] B(z, p-z),
$$

that is, for $a, b>0,0<c<a, c<\Re(p)<b+c$,

$$
\begin{aligned}
\frac{\Gamma(a+b-p)}{\Gamma(a+b)} & =\frac{1}{2 \pi i} \int_{\ell_{c}} d z \frac{\Gamma(a-z) \Gamma(b+z-p) \Gamma(z) \Gamma(p-z)}{\Gamma(a) \Gamma(b) \Gamma(p)} \\
& =\frac{1}{2 \pi i} \int_{\ell_{0}} d \zeta \frac{\Gamma(a-c-\zeta) \Gamma(b+c+\zeta-p) \Gamma(c+\zeta) \Gamma(p-c-\zeta)}{\Gamma(a) \Gamma(b) \Gamma(p)}
\end{aligned}
$$

Let $A=b+c-p, B=c, C=a-c, D=p-c$. Then the above identity reads

$$
\frac{\Gamma(A+C)}{\Gamma(A+B+C+D)}=\frac{1}{2 \pi i} \int_{\ell_{0}} d \zeta \frac{\Gamma(A+\zeta) \Gamma(B+\zeta) \Gamma(C-\zeta) \Gamma(D-\zeta)}{\Gamma(B+C) \Gamma(A+D) \Gamma(B+D)} .
$$

This is the formula that has to be proved, except that here there are restrictions: $\Re(A, D)>0, B, C>0$. The poles of $\Gamma(A+\zeta)$ and $\Gamma(B+\zeta)$ are to the left of $\ell_{0}$, and the poles of $\Gamma(C-\zeta)$ and $\Gamma(D-\zeta)$ are to the right of $\ell_{0}$. All that is needed to get Barnes' first lemma is to extend the result to any $A, B, C, D \in \mathbb{C}$ such that the contour can be drawn, by curving the path of integration if necessary. This is obvious, by Cauchy's theorem and analytic continuation. This proves that the additivity property of gamma distributions implies Barnes' first lemma; the converse is obvious.

Remark. A colleague (Felisa Vázquez-Abad) observed that, at first glance, the three original parameters $a, b, p$ appear to generate the four new parameters $A, B, C, D$. This is not actually the case, because $c$ may also be varied. The transformation $(a, b, c, p) \mapsto(A, B, C, D)$ is non-singular. $\square$

We now recall other properties of gamma variables, derived in Dufresne (1998). Proofs are summarized.

Proposition 4.2. Let $G_{j}^{(\alpha)} \sim \operatorname{Gamma}(\alpha, 1), B_{k}^{(\alpha, \beta)} \sim \operatorname{Beta}(\alpha, \beta)$, and suppose that all variables on the same side of an identity are independent.

(a) For any variable $U$ and any $a, b>0$,

$$
U G_{1}^{(a)}+G_{2}^{(b)} \stackrel{\mathrm{d}}{=} G_{3}^{(a+b)}\left[1+(U-1) B_{1}^{(a, b)}\right] .
$$

(b) For any $a, b, c, d>0$,

$$
B_{1}^{(a, b)} G_{1}^{(c)}+G_{2}^{(d)} \stackrel{\mathrm{d}}{=} G_{3}^{(c+d)}\left(1-B_{2}^{(b, a)} B_{3}^{(c, d)}\right) .
$$

(c) For any $a, b, c>0$,

$$
B_{1}^{(a, b+c)} G_{1}^{(b)}+G_{2}^{(c)} \stackrel{\mathrm{d}}{=} G_{3}^{(b+c)} B_{2}^{(a+c, b)} \stackrel{\mathrm{d}}{=} B_{3}^{(b+c, a)} G_{4}^{(a+c)} .
$$

(d) For any $a, b, c, d>0$,

$$
\frac{G_{1}^{(a)}}{B_{1}^{(c, d)}}+G_{2}^{(b)} \stackrel{\mathrm{d}}{=} G_{3}^{(a+b)}\left(\frac{B_{2}^{(a, b)}}{B_{3}^{(c, d)}}+1-B_{2}^{(a, b)}\right) \stackrel{\mathrm{d}}{=} G_{3}^{(a+b)}\left(1+B_{2}^{(a, b)} \frac{G_{4}^{(d)}}{G_{5}^{(c)}}\right) .
$$


(e) For any $a, b, c>0$,

$$
\frac{G_{1}^{(a)}}{B_{1}^{(b, a+c)}}+G_{2}^{(c)} \stackrel{\mathrm{d}}{=} \frac{G_{3}^{(a+c)}}{B_{2}^{(b, a)}}
$$

Proof. Recall the following property of gamma distributions: if $G_{1}^{(a)}$ and $G_{2}^{(b)}$ are independent, then

$$
\frac{G_{1}^{(a)}}{G_{1}^{(a)}+G_{2}^{(b)}} \text { is independent of } G_{1}^{(a)}+G_{2}^{(b)} .
$$

To prove (a), use (4.1) and $G_{3}^{(a+b)} \stackrel{\mathrm{d}}{=} G_{2}^{(a)}+G_{1}^{(b)}$ :

$$
G_{3}^{(a+b)}\left[1+(U-1) B_{1}^{(a, b)}\right] \stackrel{\mathrm{d}}{=} G_{3}^{(a+b)}\left(\frac{G_{2}^{(b)}}{G_{1}^{(a)}+G_{2}^{(b)}}+\frac{U G_{1}^{(a)}}{G_{1}^{(a)}+G_{2}^{(b)}}\right) \stackrel{\mathrm{d}}{=} G_{2}^{(b)}+U G_{1}^{(a)} .
$$

Part (b) results from (a), after noting that $1-B^{(a, b)} \stackrel{\mathrm{d}}{=} B^{(b, a)}$. To obtain (c), let $c=b^{\prime}, d=c^{\prime}, b=$ $b^{\prime}+c^{\prime}$ in (b). Then, using (4.1) (or Mellin transforms),

$$
B_{2}^{(b, a)} B_{3}^{(c, d)}=B_{2}^{\left(b^{\prime}+c^{\prime}, a\right)} B_{3}^{\left(b^{\prime}, c^{\prime}\right)} \stackrel{\mathrm{d}}{=} B_{4}^{\left(b^{\prime}, a+c^{\prime}\right)},
$$

which yields the first identity in (c). The second one is proved using Mellin transforms. Parts (d) and (e) are proved similarly.

Theorem 4.3. Barnes' second lemma is equivalent to either property (c) or property (e) of Proposition 4.2.

Proof. Write identity (c) using Theorem 3.1: if $a, b, c>0,0<c_{0}<a \wedge b, c_{0}<\Re(p)<c+c_{0}$,

$$
\begin{aligned}
\frac{1}{2 \pi i} \int_{\ell_{c_{0}}} d z \frac{\Gamma(a-z) \Gamma(b-z) \Gamma(p-z) \Gamma(c-p+z) \Gamma(z)}{\Gamma(a+b+c-z)} & \\
= & \frac{\Gamma(a) \Gamma(b) \Gamma(c) \Gamma(p)}{\Gamma(a+b+c)} \cdot \frac{\Gamma(b+c-p) \Gamma(a+c-p) \Gamma(a+b+c)}{\Gamma(b+c) \Gamma(a+c) \Gamma(a+b+c-p)} .
\end{aligned}
$$

The left-hand side is

$$
\frac{1}{2 \pi i} \int_{\ell_{0}} d \zeta \frac{\Gamma\left(a-c_{0}+\zeta\right) \Gamma\left(b-c_{0}+\zeta\right) \Gamma\left(p-c_{0}+\zeta\right) \Gamma\left(c-p+c_{0}-\zeta\right) \Gamma\left(c_{0}-\zeta\right)}{\Gamma\left(a+b+c-c_{0}+\zeta\right)} .
$$

The poles are to the right or left of the path of integration as required for Barnes' second lemma. It is possible to let $c_{0} \downarrow 0$, if at the same time the path of integration is moved to the left of the origin (which is a pole of $\Gamma(-\zeta)$ ). Letting $A=a, B=b, C=p, D=c-p$ yields Barnes' second lemma with the stated restrictions on $a, b, c$ and $p$. The general formula is obtained by analytic continuation, which is possible if the path of integration is moved so as to keep the poles of $\Gamma(A+\zeta), \Gamma(B+\zeta), \Gamma(C+\zeta)$ to the left of the path, and those of $\Gamma(D-\zeta), \Gamma(-\zeta)$ to the right of the path. This proves the equivalence of Barnes' second lemma and Theorem 4.2 (c).

Similarly, property (e) says that, for $a>c_{0}>0, b, c>0, c_{0}<\Re(p)<c+c_{0}$,

$$
\begin{aligned}
& \frac{1}{2 \pi i} \int_{\ell_{0}} d \zeta \frac{\Gamma\left(a-c_{0}-\zeta\right) \Gamma\left(b+c_{0}+\zeta\right) \Gamma\left(c-p+c_{0}+\zeta\right) \Gamma\left(p-c_{0}-\zeta\right) \Gamma\left(c_{0}+\zeta\right)}{\Gamma\left(a+b+c+c_{0}+\zeta\right)} \\
& \quad=\frac{\Gamma(a) \Gamma(c) \Gamma(p) \Gamma(a+c-p) \Gamma(b+p) \Gamma(a+b)}{\Gamma(a+b+c) \Gamma(a+c) \Gamma(a+b+p)}
\end{aligned}
$$


It is possible to let $c_{0} \rightarrow p$ in the integral, while suitably curving the path of integration. Relabelling the parameters $(A=b+p, B=p, C=c, D=a-p)$ yields Barnes' second lemma, by analytic continuation.

Parts (b) and (d) of Proposition 4.2 may also be rewritten in terms of Barnes integrals. In the first case, raise both sides to power $-p$ and take expectations; then Theorem 3.1 and an obvious expansion on the right yield:

$$
\begin{aligned}
\frac{1}{2 \pi i} \int_{\ell_{c_{0}}} d z \frac{\Gamma(a-z) \Gamma(c-z) \Gamma(d-p+z) \Gamma(z) \Gamma(p-z)}{\Gamma(a+b-z)} & \\
= & \frac{\Gamma(a) \Gamma(c) \Gamma(d) \Gamma(p) \Gamma(c+d-p)}{\Gamma(a+b) \Gamma(c+d)}{ }_{3} F_{2}(b, c, p ; a+b, c+d ; 1) .
\end{aligned}
$$

A similar formula appears in the proof of Barnes' second lemma in Andrews et al. (2000, p.91). Part (d) of Proposition 4.2 becomes an identity between integrals:

$$
\begin{aligned}
& \int_{\ell_{c_{0}}} d z \frac{\Gamma(a-z) \Gamma(c+z) \Gamma(b-p+z) \Gamma(z) \Gamma(p-z)}{\Gamma(c+d+z)} \\
&=\frac{\Gamma(b) \Gamma(a+b-p)}{\Gamma(c+d) \Gamma(d)} \int_{\ell_{c_{0}}} d s \frac{\Gamma(a-s) \Gamma(d-s) \Gamma(c+s) \Gamma(s) \Gamma(p-s)}{\Gamma(a+b-s)} .
\end{aligned}
$$

Both formulas above extend to complex $a, b, c$ by analytic continuation.

At the beginning of this section it is claimed that property (a) in Proposition 4.2 is equivalent to (4.1). How (4.1) may be obtained from Proposion 4.2 (a) will now be shown. It is sufficient to prove that

$$
\mathrm{E}\left(\frac{G_{1}^{(a)}}{G_{1}^{(a)}+G_{2}^{(b)}}\right)^{s}\left(G_{1}^{(a)}+G_{2}^{(b)}\right)^{t}=\mathrm{E}\left(\frac{G_{1}^{(a)}}{G_{1}^{(a)}+G_{2}^{(b)}}\right)^{s} \mathrm{E}\left(G_{1}^{(a)}+G_{2}^{(b)}\right)^{t}
$$

for all $s, t \in \mathbb{N}$. Raise the identity in Proposition 4.2 (a) to power $t$, take expectations on both sides, differentiate $s$ times with respect to $U$, and finally let $U=1$. The result is $t(t-1) \cdots(t-s+1)$ times

$$
\mathrm{E}\left(G_{1}^{(a)}\right)^{s}\left(G_{1}^{(a)}+G_{2}^{(b)}\right)^{t-s}=\mathrm{E}\left(G_{3}^{(a+b)}\right)^{t}\left(B_{1}^{(a, b)}\right)^{s},
$$

which is (4.2), since $B_{1}^{(a, b)}$ and $G_{3}^{(a)}$ are independent in the proposition.

\section{$5 \quad$ Properties of reciprocal gamma variables}

We begin by looking at the distribution of

$$
H^{(a, b)}=\left(\frac{1}{G_{1}^{(a)}}+\frac{1}{G_{2}^{(b)}}\right)^{-1}=\frac{G_{1}^{(a)} G_{2}^{(b)}}{G_{1}^{(a)}+G_{2}^{(b)}},
$$

where $a, b>0$ and the the two gamma variables are independent. Next, we turn to an identity for reciprocal gamma variables in Chaumont \& Yor (2003) and its consequences. 
The distribution of $H^{(a, b)}$ will turn out to be related to the beta product distribution, defined in Dufresne (2010); that paper contains the proof of the following result (Theorems 2.1 and 2.2 therein). Recall that $(\alpha)_{s}=\Gamma(\alpha+s) / \Gamma(\alpha)$.

Proposition 5.1. The Mellin transform

$$
p \mapsto \frac{(a)_{p}(c)_{p}}{(a+b)_{p}(c+d)_{p}}
$$

(in simplified form) corresponds to a probability distribution on $(0,1)$ if, and only if, the parameters satisfy: $a, c, b+d, \Re(a+b), \Re(c+d)>0$, and either

(i) (real case) b, $d$ are real and $\min (a, c)<\min (a+b, c+d)$, or

(ii) (complex case) $\operatorname{Im}(b)=-\operatorname{Im}(d) \neq 0$ and $a+b=\overline{c+d}$.

This distribution will be denoted $\operatorname{BetaProduct}(a, b, c, d)$, and " $B^{(a, b, c, d) "}$ will represent a variable with that distribution.

It can be shown (Dufresne, 2010) that the density of $B^{(a, b, c, d)}$ is

$$
\frac{\Gamma(a+b) \Gamma(c+d)}{\Gamma(a) \Gamma(c) \Gamma(b+d)} u^{a-1}(1-u)_{2}^{b+d-1} F_{1}(a+b-c, d ; b+d ; 1-u) \mathbf{1}_{\{0<u<1\}} .
$$

From the Mellin transform it is obvious that when $a, b, c, d>0$ the $\operatorname{BetaProduct}(a, b, c, d)$ is the distribution of the product of two independent variables $B_{1}^{(a, b)}$ and $B_{2}^{(c, d)}$. (N.B. The beta product distribution is studied further in Section 6.)

Theorem 5.2. (a) If $\Re(p)>-\min (a, b)$, then

$$
\mathrm{E}\left(H^{(a, b)}\right)^{p}=\frac{(a)_{p}(b)_{p}(a+b)_{p}}{(a+b)_{2 p}} .
$$

(b) For any $0<a, b<\infty$,

$$
H^{(a, b)} \stackrel{\mathrm{d}}{=} \frac{1}{4} B^{\left(a, \frac{b-a}{2}, b, \frac{a-b+1}{2}\right)} G^{(a+b)},
$$

where the variables on the right are independent. The density of $B^{\left(a, \frac{b-a}{2}, b, \frac{a-b+1}{2}\right)}$ is:

$$
2^{-a-b} \frac{\Gamma(a+b)}{\Gamma(a) \Gamma(b)} u^{a-1}(1-u)^{-\frac{1}{2}}\left[(1+\sqrt{1-u})^{b-a}+(1-\sqrt{1-u})^{b-a}\right] \mathbf{1}_{(0,1)}(u) .
$$

If $0<a \leq b \leq a+1$, then the " $B$ " above has the same distribution as the product of two independent ordinary beta variables:

$$
B^{\left(a, \frac{b-a}{2}, b, \frac{a-b+1}{2}\right)} \stackrel{\mathrm{d}}{=} B_{1}^{\left(a, \frac{b-a}{2}\right)} B_{2}^{\left(b, \frac{a-b+1}{2}\right)} .
$$

$\left(\right.$ Here $B^{(\alpha, 0)}=1$ for $\alpha>0$.) In particular, if $a>0$ then

$$
H^{(a, a)} \stackrel{\mathrm{d}}{=} \frac{1}{4} B^{\left(a, \frac{1}{2}\right)} G^{(2 a)}, \quad H^{(a, a+1)} \stackrel{\mathrm{d}}{=} \frac{1}{4} B^{\left(a, \frac{1}{2}\right)} G^{(2 a+1)} .
$$

(c) For $a, b>0$ and $\Re(s)>-4$, $\mathrm{E} e^{-s H^{(a, b)}}={ }_{3} F_{2}\left(a, b, a+b ; \frac{a+b}{2}, \frac{a+b+1}{2} ;-\frac{s}{4}\right)$. 
(d) For any $a, b>0,\left(G^{(a+b)}\right)^{2} H^{(a, b)} \stackrel{\mathrm{d}}{=} G_{1}^{(a)} G_{2}^{(b)} G_{3}^{(a+b)}$, where the variables on either side are independent.

Proof. (a) Apply Theorem 3.1 and Barnes' first lemma: for $\Re(p)>c>0$,

$$
\begin{aligned}
\mathrm{E}\left(\frac{1}{G_{1}^{(a)}}+\frac{1}{G_{2}^{(b)}}\right)^{-p} & =\frac{1}{2 \pi i} \int_{\ell_{c}} d z \frac{\Gamma(a+z) \Gamma(b+p-z)}{\Gamma(a) \Gamma(b)} \frac{\Gamma(z) \Gamma(p-z)}{\Gamma(p)} \\
& =\frac{\Gamma(a+p) \Gamma(b+p) \Gamma(a+b+p)}{\Gamma(a) \Gamma(b) \Gamma(a+b+2 p)}
\end{aligned}
$$

For part (b), note that by the duplication formula for the gamma function,

$$
\mathrm{E}\left(H^{(a, b)}\right)^{p}=\frac{(a)_{p}(b)_{p}(a+b)_{p}}{4^{p}\left(\frac{a+b}{2}\right)_{p}\left(\frac{a+b+1}{2}\right)_{p}} .
$$

The result follows from the Mellin transform of the variable $\frac{1}{4} B^{\left(a, \frac{b-a}{2}, b, \frac{a-b+1}{2}\right)} G^{(a+b)}$. It can be checked that the parameters $A=a, B=\frac{b-a}{2}, C=b, D=\frac{a-b+1}{2}$ yield a valid BetaProduct $(A, B, C, D)$ distribution:

$$
A, C, A+B, C+D, B+D>0, \quad \min (A, C)=\min (a, b)<\frac{a+b}{2}=\min (A+B, C+D)
$$

(when $a$ is equal to either $b-1, b$ or $b+1$ the beta product reduces to an ordinary beta). The density of the beta product variable is, from (5.1):

$$
\frac{\Gamma\left(\frac{a+b}{2}\right) \Gamma\left(\frac{a+b+1}{2}\right)}{\Gamma(a) \Gamma(b) \Gamma\left(\frac{1}{2}\right)} u^{a-1}(1-u)^{-\frac{1}{2}}{ }_{2} F_{1}\left(\frac{a-b}{2}, \frac{a-b+1}{2} ; \frac{1}{2} ; 1-u\right) \mathbf{1}_{\{0<u<1\}} .
$$

The duplication formula expresses the leading constant as $2^{1-a-b} \Gamma(a+b) / \Gamma(a) \Gamma(b)$. The hypergeometric function is of the form ${ }_{2} F_{1}\left(\alpha, \alpha+\frac{1}{2} ; \frac{1}{2} ; z\right)$, which is simplified as follows. First, use formula (9.5.10), p.250, in Lebedev (1972) to get

$$
\begin{aligned}
{ }_{2} F_{1}\left(\alpha, \alpha+\frac{1}{2} ; \frac{1}{2} ; z\right)= & z^{-\alpha} \frac{\Gamma\left(\frac{1}{2}\right) \Gamma(-2 \alpha)}{\Gamma\left(\frac{1}{2}-\alpha\right) \Gamma(-\alpha)}{ }_{2} F_{1}\left(\alpha, \alpha+\frac{1}{2} ; 2 \alpha+1 ; 1-\frac{1}{z}\right) \\
& +z^{\alpha-\frac{1}{2}}(1-z)^{-2 \alpha} \frac{\Gamma\left(\frac{1}{2}\right) \Gamma(2 \alpha)}{\Gamma(\alpha) \Gamma\left(\alpha+\frac{1}{2}\right)}{ }_{2} F_{1}\left(\frac{1}{2}-\alpha, 1-\alpha ; 1-2 \alpha ; 1-\frac{1}{z}\right) .
\end{aligned}
$$

Next, using the duplication formula and identities (9.8.3), p.259 in Lebedev (1972), this is equal to

$$
\begin{aligned}
& 2^{-2 \alpha-1} z^{-\alpha}\left(\frac{1+\sqrt{1 / z}}{2}\right)^{-2 \alpha}+2^{2 \alpha-1} z^{\alpha-\frac{1}{2}}(1-z)^{-2 \alpha} \sqrt{z}\left(\frac{1+\sqrt{1 / z}}{2}\right)^{2 \alpha} \\
& =\frac{1}{2}(1+\sqrt{z})^{-2 \alpha}+\frac{1}{2}(1-\sqrt{z})^{-2 \alpha},
\end{aligned}
$$

and thus the density of $B^{\left(a, \frac{b-a}{2}, b, \frac{a-b+1}{2}\right)}$ is

$$
2^{-a-b} \frac{\Gamma(a+b)}{\Gamma(a) \Gamma(b)} u^{a-1}(1-u)^{-\frac{1}{2}}\left[(1+\sqrt{1-u})^{b-a}+(1-\sqrt{1-u})^{b-a}\right] .
$$


Now turn to part (c). For $|s|<4$ we have the convergent series

$$
\mathrm{E} e^{-s H^{(a, b)}}=\sum_{n=0}^{\infty} \frac{(a)_{n}(b)_{n}(a+b)_{n}}{\left(\frac{a+b}{2}\right)_{n}\left(\frac{a+b+1}{2}\right)_{n}} \frac{(-s)^{n}}{4^{n} n !}={ }_{3} F_{2}\left(a, b, a+b ; \frac{a+b}{2}, \frac{a+b+1}{2} ;-\frac{s}{4}\right)
$$

The formula is also true for $s \geq 4$ by analytic continuation; another way to see this is to note that

$$
\mathrm{E} e^{-s H^{(a, b)}}=\int_{0}^{1} d u f_{B}(u)\left(1+\frac{s u}{4}\right)^{-a-b}
$$

where $f_{B}(\cdot)$ is the density of $B^{\left(a, \frac{b-a}{2}, b, \frac{a-b+1}{2}\right)}$. Part $(\mathrm{d})$ is obvious using Mellin transforms.

Parts of Theorem 5.2 are extended in Theorem 6.6.

Corollary 5.3. (a) The identity in law

$$
\frac{1}{G_{1}^{(a)}} \stackrel{\mathrm{d}}{=} A\left(\frac{1}{G_{2}^{(a)}}+\frac{1}{G_{3}^{(b)}}\right),
$$

with independent variables on the right, has a solution $A$ if, and only if, one of the three cases below occurs:

(i) $0<a<b<\infty, b>\frac{1}{2}$. Then

$$
A \stackrel{\mathrm{d}}{=} \frac{1}{4 B^{\left(\frac{a+b}{2}, \frac{b-a}{2}, \frac{a+b+1}{2}, \frac{a+b-1}{2}\right)}} .
$$

When $a+1 \leq b$,

$$
B^{\left(\frac{a+b}{2}, \frac{b-a}{2}, \frac{a+b+1}{2}, \frac{a+b-1}{2}\right)} \stackrel{\mathrm{d}}{=} B_{1}^{\left(\frac{a+b}{2}, \frac{a+b}{2}\right)} B_{2}^{\left(\frac{a+b+1}{2}, \frac{b-a-1}{2}\right)}
$$

when $a+b \geq 1$,

$$
B^{\left(\frac{a+b}{2}, \frac{b-a}{2}, \frac{a+b+1}{2}, \frac{a+b-1}{2}\right)} \stackrel{\mathrm{d}}{=} B_{1}^{\left(\frac{a+b}{2}, \frac{b-a}{2}\right)} B_{2}^{\left(\frac{a+b+1}{2}, \frac{a+b-1}{2}\right)} .
$$

In both cases $B_{1}$ and $B_{2}$ are independent.

(ii) $a=b>\frac{1}{2}$. Then

$$
A \stackrel{\mathrm{d}}{=} \frac{1}{4 B^{\left(a+\frac{1}{2}, a-\frac{1}{2}\right)}}
$$

(iii) $a=b=\frac{1}{2}$. Then $A=\frac{1}{4}$ and

$$
\frac{4}{G_{1}^{\left(\frac{1}{2}\right)}} \stackrel{\mathrm{d}}{=} \frac{1}{G_{2}^{\left(\frac{1}{2}\right)}}+\frac{1}{G_{3}^{\left(\frac{1}{2}\right)}}
$$

(b) In any one of the three cases above, let $G_{k}^{(b)}, A_{n}=\left(4 B_{n}^{\left(\frac{a+b}{2}, \frac{b-a}{2}, \frac{a+b+1}{2}, \frac{a+b-1}{2}\right)}\right)^{-1}, k, n=1,2, \ldots$, be independent. Then

$$
\sum_{n=1}^{\infty} A_{1} \cdots A_{n} \frac{1}{G_{n}^{(b)}} \stackrel{\mathrm{d}}{=} \frac{1}{G_{0}^{(a)}} .
$$


Proof. (a) If (5.2) holds, then necessarily $A>0$ and

$$
\mathrm{E} A^{-p}=\frac{\mathrm{E}\left(G^{(a)}\right)^{p}}{\mathrm{E}\left(H^{(a, b)}\right)^{p}}=\frac{4^{p}\left(\frac{a+b}{2}\right)_{p}\left(\frac{a+b+1}{2}\right)_{p}}{(b)_{p}(a+b)_{p}} .
$$

If $a<b$ and $b>\frac{1}{2}$, then this is $4^{p}$ times the Mellin transform of $B^{(A, B, C, D)}$, with

$$
A=\frac{a+b}{2}, \quad B=\frac{b-a}{2}, \quad C=\frac{a+b+1}{2}, \quad D=\frac{a+b-1}{2} .
$$

These are valid parameters for a beta product distribution:

$$
\begin{aligned}
& A, C, A+B, C+D>0, \quad B+D=b-\frac{1}{2}>0 \\
& A=\min (A, C)<\min (A+B, C+D)=A+B .
\end{aligned}
$$

This gives case (i). If $a=b \geq \frac{1}{2}$, then $\mathrm{E} A^{-p}$ reduces to $4^{p}$ times

$$
\frac{\left(a+\frac{1}{2}\right)_{p}}{(2 a)_{p}}
$$

which corresponds to a beta distribution (case (ii)) or to the constant 1 (case (iii)).

The assumption $a>b>0$ implies that $\frac{a+b}{2}>b$, and this leads to $\mathrm{E} A^{-b}=0$, which is impossible. If it is supposed that $a<b<\frac{1}{2}$, then the Mellin transform of $U=1 / 4 A$ is

$$
\frac{(A)_{p}(C)_{p}}{(A+B)_{p}(C+D)_{p}}
$$

with $A, C, A+B, C+D>0$ but $B+D<0$. The asymptotic formula

$$
\frac{\Gamma(\alpha+z)}{\Gamma(\beta+z)} \sim z^{\alpha-\beta}\left(1+\frac{(\alpha-\beta)(\alpha+\beta-1)}{2 z}+\mathcal{O}\left(|z|^{-2}\right)\right)
$$$$
\text { as } z \rightarrow \infty, \quad|\arg z| \leq \pi-\delta
$$

(Lebedev, 1972, p.15) implies that

$$
\mathrm{E} U^{p} \sim K p^{-B-D}
$$

as $p \rightarrow \infty, K$ a constant. Then (5.4) tends to infinity, and so the law of $U$ cannot be restricted to $[0,1]$. Moreover, applying Markov's inequality with $u_{0}>1$ gives

$$
\mathrm{P}\left(U \geq u_{0}\right)=\mathrm{P}\left(U^{p} \geq u_{0}^{p}\right) \leq 2 K u_{0}^{-p} p^{-B-D}
$$

for $p$ large enough. This tends to 0 as $p$ increases, and is therefore a contradiction. It is thus not possible that $A$ satisfies (5.2) and that $a<b<\frac{1}{2}$. The case $a=b<\frac{1}{2}$ leads to (5.3) as in cases (ii) and (iii) above, but this time $\mathrm{E}(1 / 4 A)^{p}$ equals 0 at $p=-2 a$, which is not possible.

Part (b) follows from well-known results (e.g. Vervaat, 1979) once it is checked that $\mathrm{E} \log ^{+}\left(1 / G^{(b)}\right)<\infty$ (which is obvious) and that $\mathrm{E} \log A<0$. The latter follows from (5.2), since that identity is of the form $X \stackrel{\mathrm{d}}{=} A(X+Y)$ with all variables positive,

$$
\mathrm{E} \log A=\mathrm{E} \log X-\mathrm{E} \log (X+Y)=\mathrm{E}[\log X-\log (X+Y)]
$$


The variable in square brackets is negative, and its expectation is finite.

\section{Another property of reciprocal gamma distributions}

We prove identities (5.6) and (5.7), which are from Chaumont \& Yor (2003, p.96), and also give a consequence for infinite sums of products. In the following, all variables on the same side of an equation are independent.

The independence of

$$
\frac{G_{1}^{(\alpha)}}{G_{1}^{(\alpha)}+G_{2}^{(\beta)}} \quad \text { and } \quad G_{1}^{(\alpha)}+G_{2}^{(\beta)}
$$

implies the two-dimensional identity in law

$$
\left(G_{1}^{(\alpha+\beta)} B^{(\alpha, \beta)}, G_{1}^{(\alpha+\beta)}\left(1-B^{(\alpha, \beta)}\right)\right) \stackrel{\mathrm{d}}{=}\left(G_{2}^{(\alpha)}, G_{3}^{(\beta)}\right) .
$$

This identity in turn implies another $(\alpha=a+b, \beta=b)$ :

$$
\left(\frac{1}{G_{1}^{(a+2 b)}}+\frac{1}{G_{1}^{(a+2 b)}} \frac{G_{2}^{(b)}}{G_{3}^{(a+b)}}, \frac{G_{2}^{(b)}}{G_{3}^{(a+b)}}\right) \stackrel{\mathrm{d}}{=}\left(\frac{1}{G_{4}^{(a+b)}}, \frac{G_{5}^{(b)}}{G_{4}^{(a+b)}}\right),
$$

whence, for any $X$ independent of the other variables,

$$
\frac{1}{G_{1}^{(a+2 b)}}+\left(X+\frac{1}{G_{1}^{(a+2 b)}}\right) \frac{G_{2}^{(b)}}{G_{3}^{(a+b)}} \stackrel{\mathrm{d}}{=} \frac{1}{G_{4}^{(a+b)}}\left(1+X G_{5}^{(b)}\right) .
$$

When $X \stackrel{\mathrm{d}}{=} 1 / G^{(a)},(5.6)$ becomes

$$
\frac{1}{G_{1}^{(a+2 b)}}+\left(\frac{1}{G_{4}^{(a)}}+\frac{1}{G_{1}^{(a+2 b)}}\right) \frac{G_{2}^{(b)}}{G_{3}^{(a+b)}} \stackrel{\mathrm{d}}{=} \frac{1}{G_{5}^{(a)}},
$$

which is then also

$$
\frac{1}{G_{1}^{(a+2 b)}}+\frac{1}{G_{1}^{(a+2 b)}} \frac{G_{2}^{(b)}}{G_{3}^{(a+b)}}+\frac{G_{2}^{(b)}}{G_{3}^{(a+b)}} X \stackrel{\mathrm{d}}{=} X
$$

or $A X+C \stackrel{\mathrm{d}}{=} X$, where $X$ is independent of $(A, C)$ and

$$
(A, C) \stackrel{\mathrm{d}}{=}\left(\frac{G_{2}^{(b)}}{G_{3}^{(a+b)}}, \frac{1}{G_{1}^{(a+2 b)}}\left(1+\frac{G_{2}^{(b)}}{G_{3}^{(a+b)}}\right)\right) \stackrel{\mathrm{d}}{=}\left(\frac{G_{2}^{(b)}}{G_{3}^{(a+b)}}, \frac{1}{G_{3}^{(a+b)}}\right),
$$

since $G_{2}^{(b)} / G_{3}^{(a+b)}$ is independent of $G_{2}^{(b)}+G_{3}^{(a+b)}$. We then have the identity

$$
X \stackrel{\mathrm{d}}{=} \frac{G_{2}^{(b)}}{G_{3}^{(a+b)}} X+\frac{1}{G_{3}^{(a+b)}}, \quad X \stackrel{\mathrm{d}}{=} \frac{1}{G^{(a)}} .
$$

The last identity yields the following result. 
Theorem 5.4. Let $a, b>0$ and suppose $\left(A_{n}, C_{n}\right), n=1,2, \ldots$, are i.i.d. with distribution (5.8). Then

$$
C_{1}+\sum_{n=0}^{\infty} A_{1} \cdots A_{n} C_{n+1} \stackrel{\mathrm{d}}{=} \frac{1}{G^{(a)}} .
$$

\section{Two formulas for modified Bessel functions $K$}

The modified Bessel function of the third kind $K_{\nu}(\cdot)$ (or Macdonald's function, see Lebedev, 1972, pp.108-119) is defined as:

$$
\begin{aligned}
& K_{\nu}(z)=\frac{\pi}{2} \frac{I_{-\nu}(z)-I_{\nu}(z)}{\sin \nu \pi}, \quad|\arg z|<\pi, \quad \nu \neq 0, \pm 1, \pm 2, \ldots \\
& K_{n}(z)=\lim _{\nu \rightarrow n} K_{\nu}(z), \quad n=0, \pm 1, \pm 2, \ldots,
\end{aligned}
$$

where $I_{\nu}(\cdot)$ is the modified Bessel function of the first kind. Commonly used formulas are $K_{-\nu}(z)=$ $K_{\nu}(z)$ and

$$
K_{\nu}(z)=\frac{1}{2}\left(\frac{z}{2}\right)^{-\nu} \int_{0}^{\infty} d t t^{\nu-1} e^{-t-\frac{z^{2}}{4 t}}, \quad \Re\left(z^{2}\right)>0
$$

A first formula is obtained by applying Theorem 2.1: if $\Re\left(w^{2}\right)>0$,

$$
\begin{aligned}
\mathrm{E} e^{-w^{2} /\left(4 G^{(a)}\right)} & =\frac{1}{\Gamma(a)} \int_{0}^{\infty} d x x^{a-1} e^{-x-\frac{w^{2}}{4 x}}=\frac{2}{\Gamma(a)}\left(\frac{w}{2}\right)^{a} K_{a}(w) \\
& =\frac{1}{\Gamma(a)} \frac{1}{2 \pi i} \int_{\ell_{c}} d z\left(\frac{w^{2}}{4}\right)^{-z} \Gamma(a+z) \Gamma(z)
\end{aligned}
$$

This was found for $a>c>0$, but analytic continuation gives:

Proposition 5.5. If $\Re\left(w^{2}\right)>0$ and $\nu \in \mathbb{C}$, then

$$
K_{\nu}(w)=\frac{1}{2}\left(\frac{w}{2}\right)^{-\nu} \frac{1}{2 \pi i} \int_{-i \infty}^{i \infty} d z\left(\frac{w^{2}}{4}\right)^{-z} \Gamma(z+\nu) \Gamma(z)
$$

where the path of integration lies to the right of the poles of $\Gamma(\nu+z) \Gamma(z)$.

This Barnes integral for the function $K_{\nu}(w)$ may also be seen as the inverse of the Mellin transform (Erdélyi et al., 1953, p.51)

$$
\int_{0}^{\infty} d t K_{\nu}(t) t^{z-1}=2^{z-2} \Gamma\left(\frac{z}{2}+\frac{\nu}{2}\right) \Gamma\left(\frac{z}{2}-\frac{\nu}{2}\right), \quad \Re(z \pm \nu)>0 .
$$

It can be checked directly that the right hand side of (5.10) is unchanged if $\nu$ is replaced with $-\nu$. Moreover, the integral representation (5.10) may be reconciled with the definition of Macdonald's function (5.9) by summing the residues. Form a closed semi-circle to the left to the origin, together with a vertical segment to the right of the origin. As the semi-circle gets larger, the integral over the circular part tends to 0 (use Corollary 1.4.3 and the same ideas as in the proof of Theorem 
2.4.1 in Askey et al. (2000, p.21)). If $\nu \notin \mathbb{N}$, then the residue theorem says that the right hand side of the formula in Theorem 5.3 equals $\Sigma_{1}+\Sigma_{2}$, with:

$$
\begin{aligned}
\Sigma_{1} & =\frac{1}{2}\left(\frac{w}{2}\right)^{-\nu} \sum_{n=0}^{\infty}\left(\frac{w^{2}}{4}\right)^{n} \frac{(-1)^{n} \Gamma(\nu-n)}{n !} \\
& =\frac{\pi}{2 \sin \pi \nu}\left(\frac{w}{2}\right)^{-\nu} \sum_{n=0}^{\infty}\left(\frac{w^{2}}{4}\right)^{n} \frac{1}{n ! \Gamma(1-\nu+n)}=\frac{\pi}{2 \sin \pi \nu} I_{-\nu}(w) \\
\Sigma_{2} & =\frac{1}{2}\left(\frac{w}{2}\right)^{-\nu} \sum_{n=0}^{\infty}\left(\frac{w^{2}}{4}\right)^{n+\nu} \frac{(-1)^{n} \Gamma(-\nu-n)}{n !} \\
& =-\frac{\pi}{2 \sin \pi \nu}\left(\frac{w}{2}\right)^{\nu} \sum_{n=0}^{\infty}\left(\frac{w^{2}}{4}\right)^{n} \frac{1}{n ! \Gamma(1+\nu+n)}=\frac{\pi}{2 \sin \pi \nu} I_{\nu}(w) .
\end{aligned}
$$

Here the reflection formula for the gamma function was used. We have thus reobtained the usual definition (5.9). The case $\nu \in \mathbb{N}$ is handled in the same way, except that the integrand has double poles at $-\nu-k, k=0,1, \ldots$

Another formula may be found from Theorem 5.2, part (b): for any $0<a, b<\infty$,

$$
\frac{1}{G_{1}^{(a)}}+\frac{1}{G_{2}^{(b)}} \stackrel{\mathrm{d}}{=} \frac{4}{B^{\left(a, \frac{b-a}{2}, b, \frac{a-b+1}{2}\right)} G_{3}^{(a+b)}},
$$

where the variables on either sides are independent. Taking Laplace transforms on both sides yields:

$$
\mathrm{E} \exp \left[-\frac{w^{2}}{4}\left(\frac{1}{G_{1}^{(a)}}+\frac{1}{G_{2}^{(b)}}\right)\right]=\int_{0}^{1} d u f_{B}(u) \mathrm{E} \exp \left(-\frac{w^{2}}{u} \frac{1}{G_{3}^{(a+b)}}\right) .
$$

Here $\Re\left(w^{2}\right)>0$ and $f_{B}(\cdot)$ is the density of $B$. The left hand side is:

$$
\frac{4}{\Gamma(a) \Gamma(b)}\left(\frac{w}{2}\right)^{a+b} K_{a}(w) K_{b}(w)
$$

while the right hand side is:

$$
\frac{2 w^{a+b}}{\Gamma(a+b)} \int_{0}^{1} d u f_{B}(u) u^{-\frac{a+b}{2}} K_{a+b}\left(\frac{2 w}{\sqrt{u}}\right) .
$$

We then have the identity $\left(\Re\left(w^{2}\right)>0\right)$

$$
2 K_{a}(w) K_{b}(w)=\int_{0}^{1} d u u^{\frac{a-b}{2}-1}(1-u)^{-\frac{1}{2}}\left[(1+\sqrt{1-u})^{b-a}+(1-\sqrt{1-u})^{b-a}\right] K_{a+b}\left(\frac{2 w}{\sqrt{u}}\right) .
$$

This extends to $a, b \in \mathbb{C}$ by analytic continuation (use the asymptotic formula in Lebedev, 1972, p.123). This formula is the same as (Lebedev, 1972, p.140):

$$
K_{\mu}(w) K_{\nu}(w)=2 \int_{0}^{\infty} d t K_{\mu+\nu}(2 w \cosh t) \cosh ((\mu-\nu) t)
$$

The equivalence of the two identities is checked by letting $\cosh t=1 / \sqrt{u}$ in the last integral. 


\section{Properties of $\mathrm{G}$ distributions}

A class of probability distributions consists of those distributions on $\mathbb{R}_{+}$that have a Mellin transform equal to

$$
\begin{aligned}
& \frac{\Gamma\left(a_{1}+p\right) \cdots \Gamma\left(a_{N_{1}}+p\right)}{\Gamma\left(a_{1}\right) \cdots \Gamma\left(a_{N_{1}}\right)} \times \frac{\Gamma\left(b_{1}\right) \cdots \Gamma\left(b_{N_{2}}\right)}{\Gamma\left(b_{1}+p\right) \cdots \Gamma\left(b_{N_{2}}+p\right)} \\
& \times \frac{\Gamma\left(c_{1}-p\right) \cdots \Gamma\left(c_{N_{3}}-p\right)}{\Gamma\left(c_{1}\right) \cdots \Gamma\left(c_{N_{3}}\right)} \times \frac{\Gamma\left(d_{1}\right) \cdots \Gamma\left(d_{N_{4}}\right)}{\Gamma\left(d_{1}-p\right) \cdots \Gamma\left(d_{N_{4}}-p\right)}
\end{aligned}
$$

for (real or complex) constants $a_{j}, b_{k}, c_{\ell}, d_{m}$. This class of distributions has been studied before, see Chamayou \& Letac (1999), Dufresne (1998) and references therein. This class is itself contained in the "H distributions" considered by Mathai \& Saxena (1973), Springer (1973), Kaluszka \& Krysicki (1997) and others. It appears that previous authors disregarded the cases where some of the parameters may be complex. The class of distributions with Mellin transforms given by (6.1) will be called the "G distributions". The name comes from Meijer's G functions, which are defined as

$$
G_{p, q}^{m, n}\left(\begin{array}{c}
\alpha_{1}, \ldots, \alpha_{p} \\
\beta_{1}, \ldots, \beta_{q}
\end{array} \mid z\right)=\frac{1}{2 \pi i} \int_{L} \frac{\prod_{j=1}^{m} \Gamma\left(\beta_{j}-s\right) \prod_{j=1}^{n} \Gamma\left(1-\alpha_{j}+s\right)}{\prod_{j=m+1}^{q} \Gamma\left(1-\beta_{j}+s\right) \prod_{j=n+1}^{p} \Gamma\left(\alpha_{j}-s\right)} z^{s} d s .
$$

A continuous probability distribution with Mellin transform (6.1) has a density which is a constant times a $\mathrm{G}$ function.

It is obvious that beta and gamma distributions, as well as the product convolution of any number of beta an gamma distributions, are all $\mathrm{G}$ distributions. The beta product distribution, defined in Section 5, is also a $\mathrm{G}$ distribution. In Theorems 4.2 and 5.2, it was seen that the following identities in distribution imply that the left hand sides are $\mathrm{G}$ distributions, though that would otherwise not have been obvious:

$$
\begin{aligned}
& B_{1}^{(a, b+c)} G_{1}^{(b)}+G_{2}^{(c)} \stackrel{\mathrm{d}}{=} G_{3}^{(b+c)} B_{2}^{(a+c, b)} \\
& \frac{G_{1}^{(a)}}{B_{1}^{(b, a+c)}+G_{2}^{(c)}} \stackrel{\stackrel{\mathrm{d}}{=} \frac{G_{3}^{(a+c)}}{B_{2}^{(b, a)}}}{ } \\
& \frac{G_{1}^{(a)} G_{1}^{(a)}}{G_{1}^{(a)}+G_{1}^{(a)}} \stackrel{\mathrm{d}}{=} \frac{1}{4} B^{\left(a, \frac{b-a}{2}, b, \frac{a-b+1}{2}\right)} G^{(a+b)} .
\end{aligned}
$$

In this section we focus on the cases where, in (6.1), $N_{3}=N_{4}=0$ and the Mellin transform is finite for all $p \geq 0$. In other words, we restrict our attention to $\mathrm{G}$ distributions $\mu$ on $(0, \infty)$ that satisfy

$$
\int_{\mathbb{R}_{+}} x^{p} \mu(d x)=\frac{\left(a_{1}\right)_{p} \cdots\left(a_{K}\right)_{p}}{\left(b_{1}\right)_{p} \cdots\left(b_{N}\right)_{p}}, \quad p \geq 0 .
$$

The distribution $\mu$ will be denoted " $\mathbf{G}\left(a_{1}, \ldots, a_{K} ; b_{1}, \ldots, b_{N}\right)$ ". The distribution of minus one times the logarithm of a variable that has a $\mathrm{G}$ distribution will be called "log G":

$$
X \sim \log \mathbf{G}(\mathbf{a} ; \mathbf{b}) \Longleftrightarrow e^{-X} \sim \mathbf{G}(\mathbf{a} ; \mathbf{b})
$$

(where $\mathbf{a}, \mathbf{b}$ are vectors of complex constants). All rational distributions on $\mathbb{R}_{+}$(Cox, 1955) are log $\mathrm{G}$ distributions. This is because the defining property of rational distributions is that their Laplace transform is a ratio of polynomials:

$$
\int e^{-p x} \nu(d x)=\frac{P(p)}{Q(p)}=C \frac{\prod_{n=1}^{N}\left(b_{n}+p\right)}{\prod_{k=1}^{K}\left(a_{k}+p\right)}=C \prod_{n=1}^{N} \frac{\Gamma\left(b_{n}+1+p\right)}{\Gamma\left(b_{n}+p\right)} \prod_{k=1}^{K} \frac{\Gamma\left(a_{k}+p\right)}{\Gamma\left(a_{k}+1+p\right)},
$$


where $C$ is a constant.

Proposition 5.1 will be extended to cases where $b+d=0$ (Theorem 6.2), and a product representation is given for distributions satisfying (6.2) with $K=N$ (Theorem 6.3). This will lead to conditions for $\log \mathrm{G}$ distributions to be infinitely divisible (Theorem 6.4). Next, the distributions $H^{(a, b)}$ of Section 5 are extended to the ratios of the product to the sum of gamma variables, and some properties of those ratios are derived (Theorem 6.5). Finally, some facts are established regarding $\mathbf{G}\left(a_{1}, \ldots, a_{K} ; b_{1}, \ldots, b_{N}\right)$ distributions with $K>N$; in particular, it will be seen that gamma products with complex parameters do exist.

The first result restricts the possible values of $K$ and $N$ in (6.2). Parts (a) and (b) extend claims (1) and (2) in Chamayou \& Letac (1999, p.1047) to cases where the parameters may be complex, with different proofs.

Theorem 6.1. Suppose $a_{k}, b_{n}$ are complex numbers, $X>0$ and

$$
\mathrm{E} X^{p}=\frac{\left(a_{1}\right)_{p} \cdots\left(a_{K}\right)_{p}}{\left(b_{1}\right)_{p} \cdots\left(b_{N}\right)_{p}}, \quad p \geq 0
$$

in simplified form (i.e. there is no $(k, n)$ such that $\left.a_{k}=b_{n}\right)$.

(a) The $\left\{a_{k}\right\}$ are either real or come in conjugate pairs; the same holds for the $\left\{b_{n}\right\}$. For all $k$, $\Re\left(a_{k}\right)>0$; the $a_{k}$ with the smallest real part, call it $a^{*}$, is real. If some $b_{n}$ is real then $a^{*}<b_{n}$.

(b) No probability distribution on $[0, \infty)$ has Mellin transform (6.2) with $K<N$.

(c) If $K=N$, then $X \in(0,1]$ and $\sum_{k}\left(a_{k}-b_{k}\right) \in(-\infty, 0]$. In those cases, the law of $X$ has a point mass at $x=1$ if, and only if, $\sum_{k} a_{k}=\sum b_{n}$, in which case $\sum_{n} a_{n}^{2} \geq \sum_{n} b_{n}^{2}$, and the point mass is

$$
m=\prod_{n=1}^{N} \frac{\Gamma\left(b_{n}\right)}{\Gamma\left(a_{n}\right)} .
$$

Proof. (a) If $g(p)=\mathrm{E} X^{p}$, then $\overline{g(\bar{p})}=g(p)$ for any $p$ to the right of the imaginary axis. This property also holds for any analytic continuation that $g$ may have in the rest of $\mathbb{C}$, and thus the zeros and poles of $g$ must be symmetrical with respect to the real axis. If there were an $a_{k}$ with a non-positive real part then the function $\mathrm{E}^{p}$ would have a pole at a $p$ with $\Re(p) \geq 0$, but this contradicts

$$
\left|\mathrm{E} X^{p}\right| \leq \mathrm{E} X^{\Re(p)}<\infty .
$$

To prove that the $a_{k}$ with the smallest real part must be real, first observe that $\mathrm{E} X^{p}$ is the Laplace transform of the distribution of $Y=-\log X$. If a Laplace transform exists for $\Re(p)>p_{0}$ but does not exist for $\Re(p)<p_{0}$ (i.e. if $p_{0}$ is an "abscissa of convergence" of the transform) then $p_{0}$ must be a singularity of the transform and it also must be real (Widder, 1941, p. 59). The analytic continuation of the Laplace transform of $Y$ has poles at $-a_{k}-j, j=0,1,2, \ldots, k=1, \ldots, K$, which means that the singularity with the largest real part (which must then be real) is $p_{0}=-a^{*}$.

The analytic continuation of the Laplace transform of $Y$ has zeros at $-b_{n}-j, j=0,1,2, \ldots, n=$ $1, \ldots, N$, . It is not possible that there be a real zero of that transform to the right of $p_{0}$, in other words if $b_{n}$ is real then necessarily $-b_{n}<p_{0}=-a^{*}$. 
(b) Suppose $K<N$. The asymptotic relation (5.5) for ratios or gamma functions gives

$$
\prod_{k=1}^{K} \frac{\Gamma\left(a_{k}+p\right)}{\Gamma\left(b_{k}+p\right)} \sim p^{a_{1}+\cdots a_{K}-b_{1}+\cdots b_{K}} .
$$

as $p \rightarrow \infty$. By Markov's inequality,

$$
\mathrm{P}\left(X \geq x_{0}\right) \leq \frac{\mathrm{E} X^{p}}{x_{0}^{p}} \sim \frac{K_{1} p^{K_{2}}}{x_{0}^{p} \Gamma\left(b_{K+1}+p\right) \cdots \Gamma\left(b_{N}+p\right)} \rightarrow 0, \quad p \rightarrow \infty
$$

(where $K_{1}, K_{2}$ are constants), for any $x_{0}>0$.

(c) The same arguments yield

$$
\mathrm{E} X^{p} \sim p^{\Sigma\left(a_{n}-b_{n}\right)} \prod_{n=1}^{N} \frac{\Gamma\left(b_{n}\right)}{\Gamma\left(a_{n}\right)}, \quad p \rightarrow \infty
$$

We already know that the $a_{k}$ (resp. $b_{n}$ ) are either real or conjugate pairs, so $q=\sum_{n}\left(a_{n}-b_{n}\right) \in \mathbb{R}$. If $q>0$, then $\mathrm{EX}^{p}$ tends to infinity at the same rate as $p^{q}$, implying that (1) the law of $X$ is not restricted to $[0,1]$, and (2) for any $x_{0}>1$,

$$
\mathrm{P}\left(X \geq x_{0}\right) \leq \frac{\mathrm{E} X^{p}}{x_{0}^{p}} \leq \frac{K_{3} p^{q}}{x_{0}^{p}}
$$

for $p$ large enough and a constant $K_{3}>0$. This is a contradiction, since $p^{q} x_{0}^{-p} \rightarrow 0$ as $p \rightarrow \infty$. Thus $q \leq 0$ and $X \leq 1$. If $q=0$, then (6.3) says that there is a probablility mass at $x=1$, equal to $m$. Moreover, (5.5) shows that, as $p \rightarrow \infty$,

$$
\begin{aligned}
\mathrm{E} X^{p} & =m+\mathrm{E} X^{p} \mathbf{1}_{\{X<1\}} \\
& \sim m\left(1+\sum_{n=1}^{N} \frac{\left(a_{n}-b_{n}\right)\left(a_{n}+b_{n}-1\right)}{2 p}\right)=m+\frac{m}{2 p} \sum_{n=1}^{N}\left(a_{n}^{2}-b_{n}^{2}\right) .
\end{aligned}
$$

Since $\mathrm{E} X^{p} \mathbf{1}_{\{X<1\}}$ has to be positive when $p$ is real, it follows that $\sum_{n}\left(a_{n}^{2}-b_{n}^{2}\right) \geq 0$.

The $G\left(a_{1}, a_{2} ; b_{1}, b_{2}\right)$ distribution with $a_{1}+a_{2}=b_{1}+b_{2}$

Theorem 6.1(c) extends Proposition 5.1. The latter says that the only probability distribution $\mathbf{G}\left(a_{1}, a_{2} ; b_{1}, b_{2}\right)$ on $(0,1)$ that satisfies (6.2) is the beta product distribution, defined in Section 5. Theorem $6.1(\mathrm{c})$ shows that the beta product is the only distribution on $(0, \infty)$ that satisfies (6.2) with $K=N=2$ and $a_{1}+a_{2}<b_{1}+b_{2}$. We now show that there are other $\mathbf{G}\left(a_{1}, a_{2} ; b_{1}, b_{2}\right)$ distributions but with support $(0,1]$. The only possibility remaining, assuming (6.2) holds with $K=N=2$, is that $a_{1}+a_{2}=b_{1}+b_{2}$. An example is given by Chamayou \& Letac (1999):

$$
\frac{(3)_{p}(1)_{p}}{(2)_{p}(2)_{p}}=\frac{1}{2}+\frac{1}{2} \frac{1}{1+p}
$$

is the Mellin transform of a mixed distribution with mass $\frac{1}{2}$ at $u=1$ and density $\frac{1}{2} \mathbf{1}_{(0,1)}(u)$. 
Theorem 6.2. Suppose $a_{n}, b_{n}, n=1,2$, satisfy $a_{1}, a_{2}>0, a_{1}+a_{2}=b_{1}+b_{2}$ and either (i) (real case) $a_{1} \wedge a_{2}<b_{1} \wedge b_{2}$, or (ii) (complex case) $\operatorname{Im}\left(b_{n}\right) \neq 0, b_{1}=\bar{b}_{2}$; let $a_{2} \leq a_{1}$ for simplicity. Then a variable $X$ having the probability distribution with a point mass

$$
m=\frac{\Gamma\left(b_{1}\right) \Gamma\left(b_{2}\right)}{\Gamma\left(a_{1}\right) \Gamma\left(a_{2}\right)}
$$

at $u=1$ and a continuous part with density

$$
g_{0}(u)=\frac{\Gamma\left(b_{1}\right) \Gamma\left(b_{2}\right)}{\Gamma\left(a_{1}\right) \Gamma\left(a_{2}\right)} u^{a_{1}-1}\left(b_{1}-a_{2}\right)\left(b_{2}-a_{2}\right){ }_{2} F_{1}\left(b_{1}-a_{2}+1, b_{2}-a_{2}+1 ; 2 ; 1-u\right) \mathbf{1}_{(0,1)}(u)
$$

has Mellin transform

$$
\mathrm{E} X^{p}=\frac{\left(a_{1}\right)_{p}\left(a_{2}\right)_{p}}{\left(b_{1}\right)_{p}\left(b_{2}\right)_{p}}, \quad p \geq 0 .
$$

There are no other $a_{1}, a_{2}, b_{1}, b_{2} \in \mathbb{C}$ with $a_{1}+a_{2}=b_{1}+b_{2}$ such that (6.4) holds in simplified form (i.e. $a_{k} \neq b_{n} \forall(k, n)$ ) for some $X \geq 0$.

In the complex case, if $a_{1}, a_{2}$ are fixed but $\left|\operatorname{Im}\left(b_{1}\right)\right| \rightarrow \infty$ then $\mathbf{G}\left(a_{1}, a_{2} ; b_{1}, b_{2}\right)$ converges to the Dirac mass at 0.

Proof. From Theorem 6.1, we know that $X \in(0,1], \mathrm{P}(X=1)=m$, that both $a_{1}, a_{2}$ are positive, and that $b_{1}, b_{2}$ are either both real or complex and conjugate. If $b_{1}, b_{2}$ are real then Theorem 6.1 also says that $a_{1} \wedge a_{2}<b_{1} \wedge b_{2}$.

Suppose $a_{1}, a_{2}, b_{1}, b_{2}$ satisfy all the conditions stated, in case (i) or (ii). To find the distribution of $X$ we use a limit argument. Let $f_{\epsilon}$ be the density function of the distribution $B^{\left(a_{1}, b_{1}-a_{1}, a_{2, \epsilon}, b_{2}-a_{2, \epsilon}\right)}=$ $\mathbf{G}\left(a_{1}, a_{2, \epsilon} ; b_{1}, b_{2}\right)$, where $0<a_{2, \epsilon}=a_{2}-\epsilon<a_{2}$. The density of $X$ is (from (5.1))

$$
f_{\epsilon}(u)=\frac{\Gamma\left(b_{1}\right) \Gamma\left(b_{2}\right)}{\Gamma\left(a_{1}\right) \Gamma\left(a_{2, \epsilon}\right) \Gamma(\epsilon)} u^{a_{1}-1}(1-u)^{\epsilon-1}{ }_{2} F_{1}\left(b_{1}-a_{2, \epsilon}, b_{2}-a_{2, \epsilon} ; \epsilon ; 1-u\right) \mathbf{1}_{(0,1)}(u) .
$$

Expand the ${ }_{2} F_{1}$ as an infinite series; the first term, integrated over $(0, v), 0<v<1$, becomes

$$
\frac{\Gamma\left(b_{1}\right) \Gamma\left(b_{2}\right)}{\Gamma\left(a_{1}\right) \Gamma\left(a_{2, \epsilon}\right) \Gamma(\epsilon)} \int_{0}^{v} d u u^{a_{1}-1}(1-u)^{\epsilon-1} \rightarrow 0
$$

as $\epsilon \downarrow 0$. For $v=1$, however, the same limit is

$$
\frac{\Gamma\left(b_{1}\right) \Gamma\left(b_{2}\right)}{\Gamma\left(a_{1}\right) \Gamma\left(a_{2, \epsilon}\right) \Gamma(\epsilon)} \int_{0}^{1} d u u^{a_{1}-1}(1-u)^{\epsilon-1}=\frac{\Gamma\left(b_{1}\right) \Gamma\left(b_{2}\right)}{\Gamma\left(a_{1}+\epsilon\right) \Gamma\left(a_{2, \epsilon}\right)} \rightarrow \frac{\Gamma\left(b_{1}\right) \Gamma\left(b_{2}\right)}{\Gamma\left(a_{1}\right) \Gamma\left(a_{2}\right)}=m
$$

as $\epsilon \downarrow 0$. Hence, in the limit the first term of the series contributes a point mass $m$ at $v=1$. The remainder of $(6.5)$ is a function $g_{\epsilon}(u)$ which is non-negative for $0<u<1$, since

$$
\frac{\left(b_{1}-a_{2, \epsilon}\right)_{n}\left(b_{2}-a_{2, \epsilon}\right)_{n}}{(\epsilon)_{n}} \geq 0,
$$

whether $b_{1}, b_{2}$ are real or complex (cases (i) or (ii) of the theorem). Then:

$$
\begin{aligned}
g_{\epsilon}(u) & =\frac{\Gamma\left(b_{1}\right) \Gamma\left(b_{2}\right)}{\Gamma\left(a_{1}\right) \Gamma\left(a_{2, \epsilon}\right)} u^{a_{1}-1} \sum_{n=1}^{\infty} \frac{\left(b_{1}-a_{2, \epsilon}\right)_{n}\left(b_{2}-a_{2, \epsilon}\right)_{n}}{\Gamma(\epsilon+n)} \frac{(1-u)^{\epsilon+n-1}}{n !} \\
& \rightarrow \frac{\Gamma\left(b_{1}\right) \Gamma\left(b_{2}\right)}{\Gamma\left(a_{1}\right) \Gamma\left(a_{2}\right)} u^{a_{1}-1} \sum_{n=1}^{\infty} \frac{\left(b_{1}-a_{2}\right)_{n}\left(b_{2}-a_{2}\right)_{n}}{\Gamma(n)} \frac{(1-u)^{n-1}}{n !} \\
& =\frac{\Gamma\left(b_{1}\right) \Gamma\left(b_{2}\right)}{\Gamma\left(a_{1}\right) \Gamma\left(a_{2}\right)} u^{a_{1}-1}\left(b_{1}-a_{2}\right)\left(b_{2}-a_{2}\right)_{2} F_{1}\left(b_{1}-a_{2}+1, b_{2}-a_{2}+1 ; 2 ; 1-u\right) \\
& =g_{0}(u) .
\end{aligned}
$$


It is easy to see that

$$
\int_{0}^{v} d u g_{\epsilon}(v) \rightarrow \int_{0}^{v} d u g_{0}(v), \quad 0<v<1
$$

We have thus proved that $\mathbf{G}\left(a_{1}, a_{2, \epsilon} ; b_{1}, b_{2}\right)$ converges weakly to the probability distribution with a point mass at 1 and density $g_{0}$ on $(0,1)$.

Finally, if $\left|\operatorname{Im}\left(b_{1}\right)\right|$ tends to infinity while $a_{1}, a_{2}$ are unchanged then

$$
\mathrm{E} X=\frac{a_{1} a_{2}}{\left|b_{1}\right|^{2}}
$$

tends to 0 , which implies that $X \stackrel{\mathrm{d}}{\rightarrow} 0$.

\section{Infinite product representation of $\mathrm{G}^{(\mathrm{N}, \mathrm{N})}$ distributions}

To shorten sentences, let us call " $G^{(K, N)}$ " the class of all $\mathrm{G}\left(a_{1}, \ldots, a_{K} ; b_{1}, \ldots, b_{N}\right)$ distributions.

Theorem 6.3. Suppose

$$
\mathrm{E} X^{p}=\frac{\left(a_{1}\right)_{p} \cdots\left(a_{N}\right)_{p}}{\left(b_{1}\right)_{p} \cdots\left(b_{N}\right)_{p}}, \quad p \geq 0
$$

(the $a_{k}, b_{n}$ may be real or complex) and that

$$
p \mapsto \frac{a_{1} \cdots a_{N}}{b_{1} \cdots b_{N}} \frac{\left(b_{1}+p\right) \cdots\left(b_{N}+p\right)}{\left(a_{1}+p\right) \cdots\left(a_{N}+p\right)}
$$

is the Laplace transform of a probability distribution on $\mathbb{R}_{+}$(the latter is always true if $N=1$ or 2). Then

$$
-\log X \stackrel{\mathrm{d}}{=} \sum_{j=0}^{\infty} Y_{j}
$$

where $\left\{Y_{j}\right\}$ are independent and

$$
\mathrm{E} e^{-p Y_{j}}=\frac{\left(a_{1}+j\right) \cdots\left(a_{N}+j\right)}{\left(b_{1}+j\right) \cdots\left(b_{N}+j\right)} \frac{\left(b_{1}+j+p\right) \cdots\left(b_{N}+j+p\right)}{\left(a_{1}+j+p\right) \cdots\left(a_{N}+j+p\right)}=\frac{\mathrm{E} e^{-(p+j) Y_{0}}}{\mathrm{E} e^{-j Y_{0}}} .
$$

The distribution of $Y_{j}$ has a point mass

$$
m_{j}=\frac{\left(a_{1}+j\right) \cdots\left(a_{N}+j\right)}{\left(b_{1}+j\right) \cdots\left(b_{N}+j\right)}
$$

at the origin and also a density which is a linear combination of functions of the type

$$
y^{n-1} e^{-\left(a_{k}+j\right) y} \mathbf{1}_{(0, \infty)}(y), \quad n \in\{1,2, \ldots, N\} .
$$

Conversely, if (6.8) holds for independent variables $Y_{j} \geq 0$, then $X=\exp \left(-Y_{0}-Y_{1}-\cdots\right)$ has Mellin transform (6.6).

Proof. Recall the infinite product formula for the gamma function:

$$
\frac{1}{\Gamma(z)}=z e^{\gamma z} \prod_{j=1}^{\infty}\left(1+\frac{z}{j}\right) e^{-\frac{z}{j}}
$$


where $\gamma$ is Euler's constant. Then

$$
\begin{aligned}
(a)_{p} & =\frac{\Gamma(a+p)}{\Gamma(a)}=\frac{a}{a+p} e^{-\gamma p} \prod_{j=1}^{\infty} \frac{\left(1+\frac{a}{j}\right)}{\left(1+\frac{a+p}{j}\right)} e^{\frac{p}{j}} \\
\frac{(a)_{p}}{(b)_{p}} & =\frac{a}{a+p} \frac{b+p}{b} \prod_{j=1}^{\infty} \frac{(a+j)(b+j+p)}{(b+j)(a+j+p)}=\prod_{j=0}^{\infty} \frac{(a+j)(b+j+p)}{(b+j)(a+j+p)} \\
\mathrm{E} X^{p} & =\prod_{j=0}^{\infty} \prod_{k=1}^{N} \frac{\left(a_{k}+j\right)\left(b_{k}+j+p\right)}{\left(b_{k}+j\right)\left(a_{k}+j+p\right)}
\end{aligned}
$$

For $p \in \mathbb{R}$,

$$
\prod_{k=1}^{N} \frac{\left(b_{k}+j+p\right)}{\left(a_{k}+j+p\right)}=\frac{\mathrm{E} X^{p+j}}{\mathrm{E} X^{p+j+1}}
$$

is real, and so is

$$
\prod_{k=1}^{N} \frac{\left(a_{k}+j\right)\left(b_{k}+j+p\right)}{\left(b_{k}+j\right)\left(a_{k}+j+p\right)}=m_{k}\left(1+\frac{P(p)}{Q(p)}\right),
$$

where $P$ and $Q$ are polynomials; $Q(p)=\left(a_{1}+j+p\right) \cdots\left(a_{N}+j+p\right)$ has degree $N$, and $P$ has degree at most $N-1$. If all the $\left\{a_{k}\right\}$ are distinct, then partial fractions give

$$
\prod_{k=1}^{N} \frac{\left(b_{k}+j+p\right)}{\left(a_{k}+j+p\right)}=1+\sum_{k=1}^{N} \frac{c_{k}}{a_{k}+j+p},
$$

and this is the Laplace transform of a unit point mass at the origin plus a combination of exponential functions

$$
\sum_{k=1}^{N} c_{k} e^{-\left(a_{k}+j\right) y} \mathbf{1}_{(0, \infty)}(y) .
$$

(Some of the $a_{k}$ may be complex.) If two or more of the $a_{k}$ are identical, then terms of the form $c_{k} y^{n-1} e^{-\left(a_{k}+j\right) y}$ make their appearance.

If $N=1$, then $0<a_{1}<b_{1}$ and (6.7) is the Laplace transform of a distribution on $\mathbb{R}_{+}$(the $\log$ beta distribution). The same is true for $N=2$, see Theorem 6.4 below.

An example is given at the end of this section where (6.6) holds, but (6.7) is not the Laplace transform of a probability distribution.

\section{Infinite divisibility of $\log \mathrm{G}^{(\mathrm{N}, \mathrm{N})}$ and $\log \mathrm{H}^{\left(\mathrm{a}_{1}, \ldots, \mathrm{a}_{\mathrm{N}} ; \mathrm{K}\right)}$ distributions}

The log beta and log gamma distributions are known to be infinitely divisible, and so it is natural to ask whether this is also true of all $\log \mathrm{G}$ distributions. We first consider the case of the $\mathrm{G}^{(N, N)}$ distributions; the general case $\mathrm{G}^{(K, N)}$ with $K \geq N$ will be considered later. When $K=N$, we are led to ask whether the distribution of $Y_{k}$ in Theorem 6.3 is infinitely divisible; if it is then the law of $\log X$ necessarily is as well. Conditions under which rational distributions are infinitely divisible have been found by several authors, including Steutel (1967), Sumita \& Masuda (1987), Sharshanova (1987), Zemanian (1959, 1961), and we are not adding much to the topic. The following 
result shows that $\log X$ in Theorem 6.3 is infinitely divisible if, and only if, the distribution of $Y_{k}$ is.

Theorem 6.4. (a) If $\min _{n} \Re\left(b_{n}\right)<\min _{k} \Re\left(a_{k}\right)$ then $\log \mathbf{G}\left(a_{1}, \ldots, a_{K} ; b_{1}, \ldots, b_{N}\right)$ is not infinitely divisible.

(b) Suppose $Y_{j} \geq 0$ has Laplace transform (6.8) for all $p \geq 0$, and let $X$ satisfy (6.6) (hence $\left.X \sim \mathbf{G}\left(a_{1}, \ldots, a_{N} ; b_{1}, \ldots, b_{N}\right)\right)$. Then the distributions of $Y_{j}$ (in (6.8)) and of $\log X$ are each infinitely divisible if, and only if,

$$
\forall x \geq 0, \quad \sum_{k=1}^{N} e^{-a_{k} x} \geq \sum_{n=1}^{N} e^{-b_{n} x} .
$$

In the cases where $N=1$ or 2 , any probability distribution on $\mathbb{R}_{+}$with Laplace transform (6.6) or (6.8) (for all $p \geq 0$ ) is infinitely divisible.

(c) Either one of the following sets of conditions are sufficient for the distribution $\mathbf{G}\left(a_{1}, \ldots, a_{N} ; b_{1}, \ldots, b_{N}\right)$ to exist and for $\log \mathbf{G}\left(a_{1}, \ldots, a_{N} ; b_{1}, \ldots, b_{N}\right)$ to be infinitely divisible:

(i) $\Re\left(a_{k}\right), \Re\left(b_{n}\right)>0$ for all $k, n$, and (6.9) holds;

(ii) for all $k: a_{k}>0$ and $\Re\left(b_{k}\right) \geq \frac{1}{N} \sum_{j=1}^{N} a_{j}$; for each $b_{k} \in \mathbb{C}-\mathbb{R}$ (if any), $\bar{b}_{k}$ is also one of the $b_{1}, \ldots, b_{N}$.

Proof. (a) This is an application of Theorem 4 in Lukacs (1952): if $\phi(z)=\mathrm{E} e^{-z Y}$ is analytic in the strip $S=\{z \mid \alpha<\Re(z)<\beta\}$, and the distribution of $Y$ is infinitely divisible, then $\phi(\cdot)$ does not have any zero in $S$. Here $Y \sim \log \mathbf{G}\left(a_{1}, \ldots, a_{K} ; b_{1}, \ldots, b_{N}\right), \phi(\cdot)$ has poles at $-a_{k}-j, j=0,1, \ldots$, and zeros at $-b_{n}-j, j=0,1, \ldots$, hence $\alpha=-\min _{k} \Re\left(a_{k}\right)$ and $\beta=\infty$.

(b) The Laplace transform in (6.8) is a ratio of products of gamma functions, since

$$
\frac{b+p}{a+p}=\frac{\Gamma(a+p) \Gamma(b+1+p)}{\Gamma(a+1+p) \Gamma(b+p)} .
$$

Hence, by Theorem 6.1(a), the $\left\{a_{k}, b_{n}\right\}$ are either real or conjugate pairs, and $\Re\left(a_{k}\right)>0$. By part (a) of this theorem, if $\min _{n} \Re\left(b_{n}\right)<\min _{k} \Re\left(a_{k}\right)$ then the distribution of $Y_{j}$ (or $-\log X$ ) is not infinitely divisible. Note that $\min _{n} \Re\left(b_{n}\right)<\min _{k} \Re\left(a_{k}\right)$ violates (6.9). Suppose $\min _{n} \Re\left(b_{n}\right) \geq \min _{k} \Re\left(a_{k}\right)$ for the rest of this proof.

Suppose any common factors have been cancelled in (6.8). The Frullani identity

$$
\log \left(1+\frac{p}{\alpha}\right)=\int_{0}^{\infty} d u \frac{1-e^{-p u}}{u} e^{-\alpha u}
$$

applies whenever $\Re(p), \Re(\alpha)>0$, and

$$
\log \frac{a_{1} \cdots a_{N}}{b_{1} \cdots b_{N}} \frac{\left(b_{1}+p\right) \cdots\left(b_{N}+p\right)}{\left(a_{1}+p\right) \cdots\left(a_{N}+p\right)}=\int_{0}^{\infty} d u\left(e^{-p u}-1\right) \frac{1}{u} \sum_{k=1}^{N}\left(e^{-a_{k} u}-e^{-b_{n} u}\right) .
$$


Then, as in the proof of Theorem 6.3,

$$
\begin{aligned}
\log \mathrm{E} X^{p} & =\log \prod_{j=0}^{\infty} \prod_{k=1}^{N} \frac{\left(a_{k}+j\right)\left(b_{k}+j+p\right)}{\left(b_{k}+j\right)\left(a_{k}+j+p\right)} \\
& =\sum_{j=0}^{\infty} \int_{0}^{\infty} d u\left(e^{-p u}-1\right) \frac{1}{u} \sum_{k=1}^{N}\left(e^{-\left(a_{k}+j\right) u}-e^{-\left(b_{n}+j\right) u}\right) \\
& =\int_{0}^{\infty} d u \frac{\left(e^{-p u}-1\right)}{1-e^{-u}} \frac{1}{u} \sum_{k=1}^{N}\left(e^{-a_{k} u}-e^{-b_{n} u}\right)
\end{aligned}
$$

This is true for $\Re(p)>0$, but extends to $\Re(p)=0$ by analytic continuation. For $p=-i q, q \in \mathbb{R}$, the last expression says that the characteristic function of $-\log X$ is of the form $e^{\psi(q)}$, with

$$
\psi(q)=\int_{(0, \infty)}\left(e^{i q u}-1\right) \nu(d u)
$$

Here the signed measure $\nu$ satisfies the conditions $\int\left(u^{2} \wedge 1\right)|\nu|(d u)<\infty$. Such representations of the $\log$ of the characteristic function of a distribution are unique (e.g. Sato, 1999, Theorem 8.1 and Exercise 12.3). Hence, the distributions of $Y_{k}$ and $\log X$ are infinitely divisible if, and only if, $\nu$ is a true measure, which is the same as (6.9).

If $N=1$, then we are dealing with the ordinary log beta distribution, which is well known to be infinitely divisible. The case $N=2$ is completely described in Proposition 5.1 and Theorem 6.2. Putting those together, we see that there are two cases, either $b_{1}$ and $b_{2}$ are real, or they are both complex and conjugate. In the first case, the parameters must satisfy the following conditions: $a_{1}, a_{2}>0, a_{1}+a_{2} \leq b_{1}+b_{2}, a_{1} \wedge a_{2}<b_{1} \wedge b_{2}$. It then follows that $a_{2} \leq b_{1}+b_{2}-a_{1}$ and thus

$$
\begin{aligned}
e^{-a_{1} x}+e^{-a_{2} x}-e^{-b_{1} x}-e^{-b_{2} x} & \geq e^{-a_{1} x}+e^{-\left(b_{1}+b_{2}-a_{1}\right) x}-e^{-b_{1} x}-e^{-b_{2} x} \\
& =\left(e^{-a_{1} x}-e^{-b_{2} x}\right)\left(1-e^{-\left(b_{1}-a_{1}\right) x}\right) .
\end{aligned}
$$

Reordering the $a_{k}, b_{n}$ if necessary, we may assume that $a_{1}=a_{1} \wedge a_{2}<b_{1}=b_{1} \wedge b_{2}$, which implies that the last expression is positive for all $x>0$. In the case where the $b_{n}$ 's are not real, the conditions are: $a_{1}, a_{2}>0, b_{1}=\bar{b}_{2}, a_{1}+a_{2} \leq b_{1}+b_{2}=2 \Re\left(b_{1}\right)$. Then

$$
\begin{aligned}
e^{-a_{1} x}+e^{-a_{2} x}-e^{-b_{1} x}-e^{-b_{2} x} & =e^{-a_{1} x}+e^{-a_{2} x}-2 e^{-\Re\left(b_{1}\right) x} \cos \left(\operatorname{Im}\left(b_{1}\right) x\right) \\
& \geq e^{-a_{1} x}+e^{-a_{2} x}-2 e^{-\Re\left(b_{1}\right) x} \\
& \geq e^{-a_{1} x}+e^{-a_{2} x}-2 e^{-\frac{1}{2}\left(a_{1}+a_{2}\right) x} .
\end{aligned}
$$

This is non-negative by Jensen's inequality.

Part (c)(i) is obvious, since any expression of the form $e^{\psi(q)}$, with $\psi(q)$ given in (6.10), is the characteristic function of an infinitely divisible distribution, if $\nu$ is a measure satisfying $\int\left(u^{2} \wedge\right.$ 1) $|\nu|(d u)<\infty$. Finally, for (c)(ii) apply Jensen's inequality: under the stated conditions,

$$
\sum_{k=1}^{N} e^{-a_{k} x} \geq N \exp -\left(\frac{1}{N} \sum_{k=1}^{N} a_{k} x\right) \geq \sum_{k=1}^{N} e^{-\Re\left(b_{k}\right) x} \geq \sum_{k=1}^{N} e^{-b_{k} x} .
$$


Parts (a) and (b) of the next result generalize Theorem 5.2 and the identities

$$
B^{(a, b)} G^{(a+b)} \stackrel{\mathrm{d}}{=} G^{(a)}, \quad \frac{G_{1}^{(a)}}{G_{1}^{(a)}+G_{2}^{(b)}} \stackrel{\mathrm{d}}{=} B^{(a, b)} .
$$

Part (c) includes as a particular case the infinite divisibility of the log beta distribution.

It is easy to see that for $f(\cdot) \geq 0, r>-\alpha$,

$$
\mathrm{E}\left(G^{(\alpha)}\right)^{r} f\left(G^{(\alpha)}\right)=(\alpha)_{r} \mathrm{E} f\left(G^{(\alpha+r)}\right)
$$

this implies that if $p r_{k}>-a_{k}, p<\sum\left(a_{k}+p r_{k}\right)$, then

$$
\mathrm{E}\left[\frac{\left(G_{1}^{\left(a_{1}\right)}\right)^{r_{1}} \cdots\left(G_{N}^{\left(a_{N}\right)}\right)^{r_{N}}}{G_{1}^{\left(a_{1}\right)}+\cdots+G_{N}^{\left(a_{N}\right)}}\right]^{p}=\left(a_{1}\right)_{p r_{1}} \cdots\left(a_{N}\right)_{p r_{N}} \frac{\Gamma\left(-p+\sum\left(a_{k}+p r_{k}\right)\right)}{\Gamma\left(\sum\left(a_{k}+p r_{k}\right)\right)}
$$

Theorem 6.5. For $N \geq 2$, let $a_{k}>0,1 \leq K \leq N, \sum a_{k}=a_{1}+\cdots+a_{N}, \bar{a}=\frac{1}{K} \sum a_{k}$, and define, for independent $G_{1}^{\left(a_{1}\right)}, \ldots, G_{N}^{\left(a_{N}\right)}$,

$$
H^{\left(a_{1}, \ldots, a_{N} ; K\right)}:=\frac{G_{1}^{\left(a_{1}\right)} \cdots G_{K}^{\left(a_{K}\right)}}{G_{1}^{\left(a_{1}\right)}+\cdots+G_{N}^{\left(a_{N}\right)}} .
$$

(a) $H^{\left(a_{1}, \ldots, a_{N} ; K\right)}\left(G_{N+1}^{\left(\Sigma a_{k}\right)}\right)^{K} \stackrel{\mathrm{d}}{=} G_{1}^{\left(a_{1}\right)} \cdots G_{K}^{\left(a_{K}\right)}\left(G_{N+1}^{\left(\Sigma a_{k}\right)}\right)^{K-1}$, where $G_{N+1}^{\left(\Sigma a_{k}\right)}$ is independent of the other variables.

indent(b) $H^{\left(a_{1}, \ldots, a_{N} ; K\right)} \stackrel{\mathrm{d}}{=} K^{-K} B\left(G^{\left(\Sigma a_{k}\right)}\right)^{K-1}$, where $B \sim \mathbf{G}\left(a_{1}, \ldots, a_{K} ; \bar{a}, \bar{a}+\frac{1}{K}, \ldots, \bar{a}+\frac{K-1}{K}\right)$ is independent of $G^{\left(\Sigma a_{k}\right)}$.

(c) The distribution of $\log H^{\left(a_{1}, \ldots, a_{N} ; K\right)}$ is infinitely divisible. This includes in particular the distribution of $\log \left(\frac{1}{G^{\left(a_{1}\right)}}+\frac{1}{G^{\left(a_{2}\right)}}\right)$

Proof. (a) Setting $r_{k}=\mathbf{1}_{\{k \leq K\}}$ in (6.11) yields:

$$
\begin{aligned}
\mathrm{E}\left(H^{\left(a_{1}, \ldots, a_{N} ; K\right)}\right)^{p} & =\left(a_{1}\right)_{p} \cdots\left(a_{K}\right)_{p} \frac{\Gamma\left(\sum a_{k}+(K-1) p\right)}{\Gamma\left(\sum a_{k}+K p\right)} \\
& =\frac{\mathrm{E}\left[G_{1}^{\left(a_{1}\right)} \cdots G_{K}^{\left(a_{K}\right)}\left(G_{N+1}^{\left(\sum a_{k}\right)}\right)^{K-1}\right]^{p}}{\mathrm{E}\left(G_{N+1}^{\left(\Sigma a_{k}\right)}\right)^{K p}} .
\end{aligned}
$$

(b) From the multiplication formula for the gamma function (Eq.( $\left.c^{\prime}\right)$ in the Appendix),

$$
\begin{aligned}
\mathrm{E}\left(H^{\left(a_{1}, \ldots, a_{N} ; K\right)}\right)^{p} & =\frac{\left(a_{1}\right)_{p} \cdots\left(a_{K}\right)_{p}}{\left(\sum a_{k}\right)_{K p}}\left(\sum a_{k}\right)_{(K-1) p} \\
& =K^{-K p} \frac{\left(a_{1}\right)_{p} \cdots\left(a_{K}\right)_{p}}{(\bar{a})_{p}\left(\bar{a}+\frac{1}{K}\right)_{p} \cdots\left(\bar{a}+\frac{K-1}{K}\right)_{p}}\left(\sum a_{k}\right)_{(K-1) p} \\
& =\mathrm{E}\left[K^{-K} B\left(G^{\left(\Sigma a_{k}\right)^{K-1}}\right]^{p} .\right.
\end{aligned}
$$


The variable $B$ has a $\mathbf{G}\left(a_{1}, \ldots, a_{K} ; \bar{a}, \bar{a}+\frac{1}{K}, \ldots, \bar{a}+\frac{K-1}{K}\right)$ distribution, which exists by Theorem 6.4 (c)(ii). The latter says moreover that the $\log \mathbf{G}\left(a_{1}, \ldots, a_{K} ; \bar{a}, \bar{a}+\frac{1}{K}, \ldots, \bar{a}+\frac{K-1}{K}\right)$ distribution is infinitely divisible, which implies part (c) of Theorem 6.5, since the $\log \operatorname{Gamma}\left(\sum a_{k}\right)$ is also infinitely divisible.

There is a superficial similarity between property (a) above and the independence of

$$
G_{1}^{(a)}+G_{2}^{(b)} \quad \text { and } \quad \frac{G_{1}^{(a)}}{G_{1}^{(a)}+G_{2}^{(b)}} .
$$

The latter implies that, if variables with different subscripts are independent,

$$
G_{1}^{(a+b)} \frac{G_{2}^{(a)}}{G_{2}^{(a)}+G_{3}^{(b)}} \stackrel{\mathrm{d}}{=}\left(G_{2}^{(a)}+G_{3}^{(b)}\right) \frac{G_{2}^{(a)}}{G_{2}^{(a)}+G_{3}^{(b)}}=G_{2}^{(a)} .
$$

A quick way to connect the first expression on the left to the last one on the right is to imagine that " $G_{1}^{(a+b)}$ and $G_{2}^{(a)}+G_{3}^{(b)}$ cancel out". Similarly, property (a) may be rewritten as

$$
\left(G_{N+1}^{\left(\sum a_{k}\right)}\right)^{K} \frac{G_{1}^{\left(a_{1}\right)} \cdots G_{K}^{\left(a_{K}\right)}}{G_{1}^{\left(a_{1}\right)}+\cdots+G_{N}^{\left(a_{K}\right)}} \stackrel{\mathrm{d}}{=} G_{1}^{\left(a_{1}\right)} \cdots G_{K}^{\left(a_{K}\right)}\left(G_{N+1}^{\left(\Sigma a_{k}\right)}\right)^{K-1}
$$

(all variables independent), which may be remembered by imagining that on the left one of the $G_{N+1}^{\left(\Sigma a_{k}\right)}$ cancels with $G_{1}^{\left(a_{1}\right)}+\cdots+G_{N}^{\left(a_{N}\right)}$.

From the expression of the Mellin transform it is possible to extend the distribution of $H^{\left(a_{1}, \ldots, a_{N} ; K\right)}$ to complex $a_{k}$; see the end of this section for an example.

\section{$\mathrm{G}^{(\mathrm{K}, \mathrm{N})}$ distributions with $\mathrm{K} \geq \mathrm{N}$; gamma products with complex parameters}

The results above concern almost exclusively the $\mathbf{G}^{(K, N)}$ distributions with $K=N$. Regarding those with $K>N$, we already know that they include all products of independent $G^{(N, N)}$ and $G_{1}^{\left(a_{1}\right)} \cdots G_{K-N}^{\left(a_{K-N}\right)}$, where the $G_{j}$ are independent. It is a natural question to ask whether these are the only possibilities. The answer is no. First, products of $M$ independent gamma variables have a $\mathbf{G}\left(a_{1}, \ldots, a_{M} ;-\right)$ distribution, and this class includes cases where some of the parameters are complex; we will call distributions in this wider class "generalized gamma products". Second, the class of all products of independent $G^{(N, N)}$ and a generalized gamma product still does not exhaust all $G^{(K, N)}$ distributions with $K>N$. These two claims will be justified after the proof of Theorem 6.6.

Parts (b) and (d) of Theorem 6.6 extend the following property of beta distributions:

$$
b B^{(a, b)} \stackrel{\mathrm{d}}{=} \frac{G_{1}^{(a)}}{\frac{1}{b} G_{1}^{(a)}+\frac{1}{b} G_{2}^{(b)}} \stackrel{\mathrm{d}}{\rightarrow} G_{1}^{(a)} \quad \text { as } \quad b \rightarrow \infty
$$

(because $\frac{1}{b} G_{1}^{(a)}$ tends to 0 , and $\frac{1}{b} G_{2}^{(b)}$ tends to 1 ). Part (b) says in particular that if it is known that $\mathbf{G}\left(a_{1}, \ldots, a_{K} ; b_{1}, \ldots, b_{N}\right)$ exists, then $\mathbf{G}\left(a_{1}, \ldots, a_{K} ; b_{1}^{\prime}, \ldots, b_{M}^{\prime}\right)$ also exists, where $\left\{b_{1}^{\prime}, \ldots, b_{M}^{\prime}\right\}$ is obtained by removing some of the parameters in $\left\{b_{1}, \ldots, b_{N}\right\}$; this is proved in general when the $b_{n}$ 's that are removed are real, but in the case where they are complex more assumptions are imposed. 
Theorem 6.6. (a) All $G^{(K, N)}$ distributions with $K>N \geq 0$ have support $(0, \infty)$, and their probability density function is infinitely differentiable at each point in that set.

(b) Suppose $X \sim \mathbf{G}\left(a_{1}, \ldots, a_{K} ; b_{1}, \ldots, b_{N}\right)$ with $K \geq N \geq J, b_{1}, \ldots, b_{J} \in \mathbb{R}$. Then

$$
b_{1} \cdots b_{J} X \stackrel{\mathrm{d}}{\rightarrow} \mathbf{G}\left(a_{1}, \ldots, a_{N} ; b_{J+1}, \ldots, b_{N}\right)
$$

as $b_{n} \rightarrow \infty$ for $1 \leq n \leq J$.

(c) If $K \geq N \geq 0$ and $\Re\left(b_{n}\right)>0$ for all $n$, the characteristic function of $Y \sim \log \mathbf{G}\left(a_{1}, \ldots, a_{K} ; b_{1}, \ldots, b_{N}\right)$ is

$$
\mathrm{E} e^{i q Y}=\frac{\left(a_{1}\right)_{-i q} \cdots\left(a_{K}\right)_{-i q}}{\left(b_{1}\right)_{-i q} \cdots\left(b_{N}\right)_{-i q}}=e^{\Psi(q)}
$$

with

$$
\Psi(q)=i q\left(\sum_{n=1}^{N} \psi\left(b_{n}\right)-\sum_{k=1}^{K} \psi\left(a_{k}\right)\right)+\int_{0}^{\infty} d u \frac{\left(e^{i q u}-1-i u q\right)}{u\left(1-e^{-u}\right)}\left(\sum_{k=1}^{K} e^{-a_{k} u}-\sum_{n=1}^{N} e^{-b_{n} u}\right)
$$

where $\psi(z)=\Gamma^{\prime}(z) / \Gamma(z)$. In all cases the $\log \mathbf{G}\left(a_{1}, \ldots, a_{K} ; b_{1}, \ldots, b_{N}\right)$ distribution is infinitely divisible if, and only if,

$$
\forall x \geq 0, \quad \sum_{k=1}^{K} e^{-a_{k} x} \geq \sum_{n=1}^{N} e^{-b_{n} x} .
$$

If all $a_{k}$ are real, then $\log \mathbf{G}\left(a_{1}, \ldots, a_{K} ; b_{1}, \ldots, b_{N}\right)$ is infinitely divisible as soon as all $\Re\left(b_{n}\right)$ are large enough.

(d) The result in (b) remains true when some of the $b_{n}$ are complex for $1 \leq n \leq J$, under the following conditions: (i) $\log \mathbf{G}\left(a_{1}, \ldots, a_{K} ; b_{1}, \ldots, b_{N}\right)$ is infinitely divisible; (ii) if $b_{n}$ is complex for some $1 \leq n \leq J$, then $\bar{b}_{n}$ is also one of $b_{1}, \ldots, b_{J}$; for any conjugate pair $b_{n_{1}}, b_{n_{2}}$, the limit is taken as $\Re\left(b_{n_{1}}\right)=\Re\left(b_{n_{2}}\right) \rightarrow \infty$ while $\operatorname{Im}\left(b_{n_{1}}\right)=-\operatorname{Im}\left(b_{n_{2}}\right)$ remains fixed.

Proof. (a) Suppose $X \sim \mathbf{G}\left(a_{1}, \ldots, a_{K} ; b_{1}, \ldots, b_{N}\right)$ with $K>N$. The characteristic function of $Y=\log X$ is $\mathrm{E} X^{i u}$, and this is an integrable function of $u$ over $\mathbb{R}$, by Lemma 2.2. Hence, $Y$ has a density function

$$
f(y)=\frac{1}{2 \pi} \int_{-\infty}^{\infty} d u e^{-i u y} \mathrm{E} X^{i u},
$$

which is an analytic function of the complex variable $y$ in the strip $|\operatorname{Im}(y)|<(K-N) \frac{\pi}{2}$ ) (this is once again because, by Lemma 2.2,

$$
\left|\mathrm{E} X^{i u}\right|=\mathcal{O}\left(|u|^{\frac{1}{2}(N-K)+\sum a_{k}-\sum b_{n}} \exp \left[-(K-N) \frac{\pi|u|}{2}\right]\right)
$$

as $|u| \rightarrow \infty)$. Hence, $f(y)$ cannot be zero over any interval $\left[y_{1}, y_{2}\right] \subset(0, \infty)$, because this would imply that $f(y)$ is 0 for all $y$.

(b) Suppose $b_{1}^{\prime}>b_{1}$; if $\mathbf{G}\left(a_{1}, \ldots, a_{K} ; b_{1}, \ldots, b_{N}\right)$ exists, then its product convolution with a $G\left(b_{1} ; b_{1}^{\prime}\right)=\mathbf{B e t a}\left(b_{1}, b_{1}^{\prime}-b_{1}\right)$ is $\mathbf{G}\left(a_{1}, \ldots, a_{K} ; b_{1}^{\prime}, b_{2}, \ldots, b_{N}\right)$. Hence, for all $b_{n}^{\prime}>b_{n}, n=1, \ldots, J$, 
we know that $\mathbf{G}\left(a_{1}, \ldots, a_{K} ; b_{1}^{\prime}, \ldots, b_{J}^{\prime}, b_{J+1} \ldots, b_{N}\right)$ also exists. The Mellin transform of $b_{1} \cdots b_{J} X$ is

$$
b_{1}^{p} \cdots b_{J}^{p} \frac{\left(a_{1}\right)_{p} \cdots\left(a_{K}\right)_{p}}{\left(b_{1}\right)_{p} \cdots\left(b_{N}\right)_{p}} .
$$

By (5.5), $b_{n}^{p} /\left(b_{n}\right)_{p} \rightarrow 1$ as $b_{n} \rightarrow \infty$, and so the limit of the Mellin transform is

$$
\frac{\left(a_{1}\right)_{p} \cdots\left(a_{K}\right)_{p}}{\left(b_{J+1}\right)_{p} \cdots\left(b_{N}\right)_{p}}
$$

We only need to check that this is the Mellin transform of a probability distribution. Just repeat the same calculation with $p=i q, q \in \mathbb{R}$. The limit of the Mellin transform is a continuous function of $q$ near the origin, which implies that there is a probability distribution with (complex) Mellin transform

$$
\frac{\left(a_{1}\right)_{i q} \cdots\left(a_{K}\right)_{i q}}{\left(b_{J+1}\right)_{i q} \cdots\left(b_{N}\right)_{i q}}
$$

(This follows from the continuity theorem for characteristic functions; see for example Feller, 1971, p.508.)

(c) We know that $\Re\left(a_{k}\right)>0$ for all $k$ (Theorem 6.1). If $\Re\left(b_{n}\right) \leq 0$ then

$$
\sum_{k} e^{-a_{k} x} \geq \sum_{n} e^{-b_{n} x}
$$

cannot hold for all $x \geq 0$, and thus (Theorem $6.4(\mathrm{a}))$ the $\log \mathbf{G}\left(a_{1}, \ldots, a_{K} ; b_{1}, \ldots, b_{N}\right)$ is not infinitely divisible. Suppose $\Re\left(b_{n}\right)>0$ in what follows.

It is well known that, if $\Re(z)>0$ (see for instance Carmona et al. (1997)),

$$
\frac{\Gamma(z+i q)}{\Gamma(z)}=\exp \left(i q \psi(z)+\int_{0}^{\infty} d u \frac{\left(e^{-i q u}-1+i q u\right)}{u\left(1-e^{-u}\right)} e^{-z u}\right)
$$

where $\psi(z)=\Gamma^{\prime}(z) / \Gamma(z)$, and this implies (6.13). The arguments used in the proof of Theorem $6.4(\mathrm{~b})$ yield the result.

(d) The limit of the Mellin transform is found in the the same way as in (b). The extra assumptions are made to ensure that if $\mathbf{G}\left(a_{1}, \ldots, a_{K} ; b_{1}, \ldots, b_{N}\right)$ is a valid distribution, then so is $\mathbf{G}\left(a_{1}, \ldots, a_{K} ; b_{1}^{\prime}, \ldots, b_{J}^{\prime}, b_{J+1}, \ldots, b_{N}\right)$ when $\Re\left(b_{n}^{\prime}\right)>\Re\left(b_{n}\right), n=1, \ldots, J$. For $b_{n}^{\prime}$ complex with $\Re\left(b_{n}^{\prime}\right)>\Re\left(b_{n}\right)$ and $\operatorname{Im}\left(b_{n}^{\prime}\right)=-\operatorname{Im}\left(b_{n}\right)$,

$$
e^{-b_{n}^{\prime} u}+e^{-\bar{b}_{n}^{\prime} u} \leq e^{-b_{n} u}+e^{-\bar{b}_{n} u}
$$

for any $u$ such that the right hand side is positive. Hence, replacing $\left(b_{n}, \bar{b}_{n}\right)$ with $\left(b_{n}^{\prime}, \bar{b}_{n}^{\prime}\right)$ in $(6.13)$ yields a valid, infinitely divisible log $\mathrm{G}$ distribution.

\section{Comments and examples}

1. There are legitimate $\mathbf{G}\left(a_{1}, \ldots, a_{K} ; b_{1}, \ldots, b_{N}\right)$ distributions with $\operatorname{Im}\left(b_{n}\right) \neq 0$ and $\Re\left(b_{n}\right) \leq 0$ for at least one pair of conjugate parameters. One way to construct such an example is to start with the 
Laplace transform of a rational distribution as in (6.7). This possibility is taken into consideration in Theorems 6.1(a), 6.4(a) and 6.6(c). In those cases the condition that

$$
\sum_{k=1}^{K} e^{-a_{k} x} \geq \sum_{n=1}^{N} e^{-b_{n} x}
$$

for all $x \geq 0$ cannot hold, because in all $\mathbf{G}\left(a_{1}, \ldots, a_{K} ; b_{1}, \ldots, b_{N}\right)$ distributions $\Re\left(a_{k}\right)>0$ for all $k$ (Theorem 6.1(a)).

2. This example shows that for $\mathrm{G}^{(N, N)}$ distributions the decomposition $-\log X=Y_{0}+Y_{1}+\cdots$ given by (6.6)-(6.8) (Theorem 6.3) does not always hold; we exhibit a $\mathrm{G}^{(3,3)}$ distribution that has a different decomposition. For $k=0,1, \ldots$, define

$$
\mu_{k}(d x)=C_{k} e^{-k x}\left\{\delta_{0}(x)+\left[e^{-x}\left(e^{-x}-.1\right)\left(e^{-x}-.15\right)\right] \mathbf{1}_{\{x>0\}} d x\right\},
$$

where $\delta_{0}$ is the Dirac mass at 0 and $C_{k}$ is such that $\mu_{k}(\mathbb{R})=1$. These are signed measures, since $\mu_{k}(d x)$ is negative for $x \in(-\log .15,-\log .1)$, and are thus not probability measures. Also,

$$
\int_{0}^{\infty} e^{-p x} \mu_{0}(d x)=C_{0}\left(1+\frac{.015}{1+p}-\frac{.25}{2+p}+\frac{1}{3+p}\right)=\frac{1 \cdot 2 \cdot 3}{b_{1} b_{2} b_{3}} \frac{\left(b_{1}+p\right)\left(b_{2}+p\right)\left(b_{3}+p\right)}{(1+p)(2+p)(3+p)}
$$

where $b_{1}=1.0120, b_{2}=1.8659, b_{3}=3.8871$ to four decimal places. Then

$$
\begin{aligned}
\mu_{0} * & \mu_{1}(d x)=\frac{6}{b_{1} b_{2} b_{3}} \frac{24}{\left(b_{1}+1\right)\left(b_{2}+1\right)\left(b_{3}+1\right)} \\
& \times\left\{\delta_{0}(x)+\left[.01835 e^{-x}-(.282725+.00375 x) e^{-2 x}+(1.67438-.25 x) e^{-3 x}+.12 e^{-4 x}\right] \mathbf{1}_{\{x>0\}} d x\right\} .
\end{aligned}
$$

This, however, is a probability measure. Hence, from Theorem 6.3, if $\left\{Z_{k}\right\}$ are independent, the distribution of $Z_{k}$ being a constant times $e^{-k x} \mu_{0} * \mu_{1}(d x)$, then

$$
X=\exp -\sum_{k=0}^{\infty} Z_{k} \sim \mathbf{G}\left(1,2,3 ; b_{1}, b_{2}, b_{3}\right) .
$$

This says that the distribution of $-\log X$ is the infinite convolution

$$
\left(\mu_{0} * \mu_{1}\right) *\left(\mu_{2} * \mu_{3}\right) * \cdots .
$$

In this expression the signed measures $\mu_{n}$ are taken two by two, but this is of no importance because $\mu_{n}$ tends narrowly to $\delta_{0}$. Hence, $\left(\mu_{0} * \mu_{1}\right) * \cdots *\left(\mu_{2 n} * \mu_{2 n+1}\right) * \mu_{2 n+2} \rightarrow \log \mathbf{G}\left(1,2,3 ; b_{1}, b_{2}, b_{3}\right)$ as well. The conclusion is: what corresponds to the decomposition (6.6)-(6.8) in Theorem 6.3 is the infinite convolution of the signed measures $\mu_{0}, \mu_{1}, \ldots$, defined above, none of which is a probability distribution. The $\log \mathbf{G}\left(1,2,3 ; b_{1}, b_{2}, b_{3}\right)$ is by Theorem 6.4 not infinitely divisible. Specifically,

$$
e^{-u}+e^{-2 u}+e^{-3 u}-e^{-b_{1} u}-e^{-b_{2} u}-e^{-b_{3} u}
$$

is negative for $u \in(1.6688,2.3185)$.

3. From the decomposition of the $H^{\left(a_{1}, \ldots, a_{N} ; K\right)}$ distribution in Theorem $6.5(\mathrm{~b})$ it is possible to find cases where the law makes sense when some of the parameters are complex. For instance, take 
$a_{1}=1, a_{2}=3+i, a_{3}=3-i$. The $\mathbf{G}\left(a_{1}, a_{2}, a_{3} ; \bar{a}, \bar{a}+\frac{1}{3}, \bar{a}+\frac{2}{3}\right)$ is then a legitimate distribution, and $H^{(1,3+i, 3-i ; 3)}$ makes sense if defined as the distribution of the product of independent variables

$$
\frac{1}{27} B G^{(7)}, \quad B \sim \mathbf{G}\left(1,3+i, 3-i ; \frac{7}{3}, \frac{8}{3}, 3\right)
$$

4. It was shown in Dufresne (2010) that the product convolution of the two functions

$$
g(a, x)=\frac{x^{a_{k}-1}}{\Gamma\left(a_{k}\right)} e^{-x} \mathbf{1}_{\{x>0\}}, \quad \Re\left(a_{k}\right)>0, \quad k=1,2,
$$

cannot be a probability density function if one or both of the $a_{k}$ are complex and have a positive real part. However, complex parameters are possible for product convolutions of three or more functions $g\left(a_{k}, x\right)$. This can be seen from (6.13) above. For $K=N=3$, it is possible to find $a_{1}>0$ and $a_{2}=\bar{a}_{3}$ with $\Re\left(a_{2}\right)>a_{1}$ such that $e^{-a_{1} x}+e^{-a_{2} x}+e^{-a_{3} x} \geq 0$ for all $x>0$. For instance, take $a_{1}=1, a_{2}=3+i$. Then

$$
\gamma(p)=\left(a_{1}\right)_{p}\left(a_{2}\right)_{p}\left(a_{3}\right)_{p}
$$

is the Mellin transform of a "generalized gamma product" with one real parameter and two complex parameters, and, moreover, the log transformed distribution is infinitely divisible.

5. The question was raised in this section as to whether all $\mathrm{G}^{(K, N)}$ distributions with $K>N$ may be factored into the product convolution of independent $\mathrm{G}^{(N, N)}$ and $\mathrm{G}^{(K-N, 0)}$ distributions. Here is a counterexample: consider a $\mathbf{G}\left(a_{1}, a_{2}, a_{3} ; b_{1}\right)$ distribution with $a_{2}, a_{3}$ complex and $b_{1}$ real. This is possible from part (c) of Theorem 6.6, for instance choose $a_{1}=1, a_{2}=3+i, b_{1}=3$; then

$$
e^{-x}+2 e^{-3 x} \cos x-e^{-3 x}=e^{-x}\left[1+e^{-2 x}(2 \cos x-1)\right]>0
$$

for all $x>0$. In this case it is obviously not possible to express the $\mathbf{G}\left(a_{1}, a_{2}, a_{3} ; b_{1}\right)$ as a product of a $\mathrm{G}^{(1,1)}$ and a $\mathrm{G}^{(2,0)}$.

\section{Appendix: Probabilistic proof of the duplication and multi- plication formulas for the gamma function}

We give a probabilistic proof of the duplication formula for the gamma function, showing that it is equivalent to the identity

$$
\left(G_{1}^{(2 x)}\right)^{2} \stackrel{\mathrm{d}}{=} 4 G_{2}^{(x)} G_{3}^{\left(x+\frac{1}{2}\right)},
$$

where the variables on the right are independent. The moments of the variables on either side are the same, but they cannot be summed to find the Laplace transform; this is why we use a similar identity for beta variables (see (a) below). This simplifies the moment uniqueness problem, because the beta distribution has bounded support. Otherwise the proof only uses convergence in distribution. It should be compared with other probabilistic proofs or interpretations in Wilks (1932), Gordon (1994) and Chaumont \& Yor (2003, p.99).

Define the gamma function as the usual integral $\int_{(0, \infty)} d x x^{\alpha-1} e^{-x}$ for $\Re(\alpha)>0$, and extend it recursively to $\mathbb{C}-\{0,-1,-2, \ldots\}$.

Theorem. Suppose all variables below are independent. The following facts hold and are equivalent: 
(a) $\left(B_{1}^{(2 x, 2 y)}\right)^{2} \stackrel{\mathrm{d}}{=} B_{2}^{(x, y)} B_{3}^{\left(x+\frac{1}{2}, y\right)}$ for any $x, y>0$.

(b) $\left(G_{1}^{(2 x)}\right)^{2} \stackrel{\mathrm{d}}{=} 4 G_{2}^{(x)} G_{3}^{\left(x+\frac{1}{2}\right)}$ for any $x>0$.

(c) $(2 z)_{2 p}=4^{p}(z)_{p}\left(z+\frac{1}{2}\right)_{p}$ for any $z, p \in \mathbb{C}$.

(d) $\Gamma(2 z) \Gamma\left(\frac{1}{2}\right)=2^{2 z-1} \Gamma(z) \Gamma\left(z+\frac{1}{2}\right)$ for any $z \in \mathbb{C}$.

Proof. (a) The integral moments of the variables in the identity are

$$
\frac{(2 x)_{2 n}}{(2 x+2 y)_{2 n}} \quad \text { and } \quad \frac{(x)_{n}}{(x+y)_{n}} \frac{\left(x+\frac{1}{2}\right)_{n}}{\left(x+y+\frac{1}{2}\right)_{n}},
$$

respectively, for $n=0,1, \ldots$ These are seen to be equal, if one writes

$$
(2 z)_{2 n}=4^{n} z\left(z+\frac{1}{2}\right)(z+1) \cdots(z+n-1)\left(z+n-\frac{1}{2}\right)=4^{n}(z)_{n}\left(z+\frac{1}{2}\right)_{n} .
$$

The distributions of the variables in the identity have bounded support and identical moments, and are thus the same. This proves (a).

(a) implies (b): by (6.12),

$$
y^{2}\left(B_{1}^{(2 x, 2 y)}\right)^{2} \stackrel{\mathrm{d}}{\rightarrow} \frac{\left(G_{1}^{(2 x)}\right)^{2}}{4}
$$

as $y \rightarrow \infty$. Similarly,

$$
y^{2} B_{2}^{(x, y)} B_{3}^{\left(x+\frac{1}{2}, y\right)} \stackrel{\mathrm{d}}{\rightarrow} G_{2}^{(x)} G_{3}^{\left(x+\frac{1}{2}\right)} .
$$

Raising identity (b) to power $p$ and then taking expectations gives identity (c) for $z=x>0$; the general case follows by analytic continuation. The result in (c) includes (d), just set $z=\frac{1}{2}$. The result in (d) implies that the variables on either side of identity (a) have the same Mellin transform.

The same ideas give a partial proof of the multiplication formula for the gamma function. Suppose $B_{j}^{\left(x+\frac{j}{m}, y\right)}, j=0, \ldots, m-1$ are independent. Then the moments argument used in the proof above leads to

$$
\left(B_{m}^{(m x, m y)}\right)^{m} \stackrel{\mathrm{d}}{=} B_{0}^{(x, y)} B_{1}^{\left(x+\frac{1}{m}, y\right)} \cdots B_{m-1}^{\left(x+\frac{m-1}{m}, y\right)}
$$

for any $x, y>0$, and then the same limit argument gives

$$
\left(G_{m}^{(m x)}\right)^{m} \stackrel{\mathrm{d}}{=} m^{m} G_{0}^{(x)} \cdots G_{m-1}^{\left(x+\frac{m-1}{m}\right)}
$$

where the variables on the right are independent. Then

$$
(m x)_{m p}=m^{m p}(x)_{p} \cdots\left(x+\frac{m-1}{m}\right)_{p}
$$

follows, and extends to $x$ and $p$ complex. Setting $x=\frac{1}{m}$ yields

$$
\Gamma(m z) \Gamma\left(\frac{1}{m}\right) \cdots \Gamma\left(\frac{m-1}{m}\right)=m^{m z-1} \Gamma(z) \Gamma\left(z+\frac{1}{m}\right) \cdots \Gamma\left(z+\frac{m-1}{m}\right) .
$$

We have then proved that $\left(a^{\prime}\right),\left(b^{\prime}\right),\left(c^{\prime}\right)$ and $\left(d^{\prime}\right)$ are equivalent. What is missing in order to prove the Gauss multiplication formula (Askey et al., 2000, pp.23-25),

$$
\Gamma(m z)(2 \pi)^{\frac{m-1}{2}}=m^{m z-\frac{1}{2}} \Gamma(z) \Gamma\left(z+\frac{1}{m}\right) \cdots \Gamma\left(z+\frac{m-1}{m}\right),
$$


is that

$$
\Gamma\left(\frac{1}{m}\right) \cdots \Gamma\left(\frac{m-1}{m}\right)=\frac{(2 \pi)^{\frac{m-1}{2}}}{\sqrt{m}} .
$$

For $m=2$ there are well-known simple probabilistic arguments to do this; Gordon (1994, Theorem 6 ) proves the general case $m \geq 2$.

\section{References}

Andrews, G.E., Askey, R., and Roy, R. (2000). Special Functions. Cambridge University Press.

Barnes, E.W. (1908). A new development of the theory of the hypergeometric functions. Proc. London Math. Soc. 6: 141-177.

Barnes, E.W. (1910). A transformation of generalized hypergeometric series. Quart. J. Math. 41: 136-140.

Bondesson, L. (1992). Generalized Gamma Convolutions and Related Classes of Distributions and Densities. Lecture notes in Statistics no.76, Springer-Verlag, New York.

Carmona, P., Petit, F., and Yor, M. (1997). On the distribution and asymptotic results for exponential functionals of Lévy processes. In Exponential Functionals and Principal Values Associated to Brownian Motion, Ed. M. Yor, Revista Matematica Iberoamericana.

Chamayou, J.-F., and Letac, G. (1999). Additive properties of the Dufresne laws and their multivariate extension. J. Theoret. Probab. 12: 1045-1066.

Chaumont, L., and Yor, M. (2003). Exercises in Probability. Cambridge University Press.

Cox, D.R. (1955). A use of complex probabilities in the theory of stochastic processes. Proc. Cambridge Philos. Soc. 51: 313-319.

Dufresne, D. (1998). Algebraic properties of beta and gamma distributions, and applications. Advances in Applied Mathematics 20: 285-299.

Dufresne, D. (2010). Beta products with complex parameters. Comm. in Stat., Theo. and Meth. 39: $837-854$.

Erdélyi, A. (1953). Higher transcendental functions, Vol.2. McGraw-Hill, New York.

Feller, W. (1971). An Introduction to Probability Theory and its Applications, Vol. 2, 2nd Ed. Wiley, New York.

Gordon, L. (1994). A stochastic approach to the gamma function. The Amer. Math. Monthly 101: 858-865.

Kaluszka, M., and Krysicki, W. (1997). On decompositions of some random variables. Metrika 46: 159-175.

Lebedev, N.N. (1972). Special Functions and their Applications. Dover, New York.

Lukacs, E., and Szasz, O. (1952). On analytic characteristic functions. Pacific J. Math. 2: 615-625.

Mathai, A.M., and Saxena, R.X. (1973). Generalized Hypergeometric Functions with Applications in Statistical and Physical Sciences. Lecture Notes in Mathematics No. 348, Springer-Verlag, Heidelberg and New York. 
Patterson, S.J. (1995). Introduction to the Theory of the Riemann Zeta Function. Cambridge Studies in Advanced Mathematics No.14, Cambridge University Press.

Sato, K. (1999). Lévy Processes and Infinitely Divisible Distributions. Cambridge Studies in Advanced Mathematics no. 68, Cambridge University Press.

Sharshanova, A.G. (1987). Meromorphic characteristic functions with three poles that are not all imaginary. Ukrainian Math. J. 39: 305-308.

Springer, M.D. (1979). The Algebra of Random Variables. John Wiley \& Sons, New York.

Steutel, F.W. (1967). Note on the infinite divisibility of exponential mixtures. Ann. Math. Statist. 38: 1303-1305.

Sumita, O., and Masuda, Y. (1987). Classes of probability density functions having Laplace transforms with negative zeros and poles. Adv. Appl. Prob. 19: 632-651.

Vervaat, W. (1979). On a stochastic difference equation and a representation of non negative infinitely divisible random variables Adv. Appl. Probab. 11: 750783.

Widder, D. V. (1941). The Laplace Transform. Princeton University Press.

Wilks, S.S. (1932). Certain generalizations in analysis of variance. Biometrika 24: 471-484.

Zemanian, A.H. (1959). On the pole and zero location of rational Laplace transformation of nonnegative functions. Proc. Amer. Math. Soc. 10: 868-872.

Zemanian, A.H. (1961). On the pole and zero location of rational Laplace transformation of nonnegative functions, II. Proc. Amer. Math. Soc. 12: 870-874. 